\title{
THERMODYNAMICS OF THE DOUBLE EXCHANGE SYSTEMS
}

\author{
Nobuo Furukawa \\ Dept. of Physics, Aoyama Gakuin University, \\ Setagaya, Tokyo 157-8572, Japan
}

\begin{abstract}
This article gives a comprehensive review on the recent studies of the double exchange systems using non-perturbative approaches; the dynamical mean-field theory and the Monte Carlo method. Investigations beyond mean-field type treatments are described. Taking into account strong spin fluctuations which create large changes in conduction electron structure, finite temperature properties as well as dynamics of the system are calculated. Comparisons with experimental data for colossal magnetoresistance manganites are made. We show that high Curie temperature $\left(T_{\mathrm{c}}\right)$ compounds, e.g. ( $\left.\mathrm{La}, \mathrm{Sr}\right) \mathrm{MnO}_{3}$, are canonical double-exchange systems. Properties of other compounds with lower $T_{\mathrm{c}}$ are discussed in relation to inhomogeneities of the system including the issue of phase separation.
\end{abstract}

\section{INTRODUCTION}

The concept of the double-exchange (DE) interaction was introduced by Zener $b$ in order to explain the ferromagnetism of the perovskite manganites $\mathrm{AMnO}_{3}$. He considered a Kondo-lattice type model

$$
\mathcal{H}=-\sum_{i j, \sigma} t_{i j}\left(c_{i \sigma}^{\dagger} c_{j \sigma}+\text { h.c. }\right)-J_{\mathrm{H}} \sum_{i} \vec{\sigma}_{i} \cdot \vec{S}_{i},
$$

where $t$ and $J_{\mathrm{H}}>0$ are $e_{\mathrm{g}}$ electron's hopping and (ferromagnetic) Hund's coupling between $e_{\mathrm{g}}$ and $t_{2 \mathrm{~g}}$ electrons, respectively. An effective Hamiltonian in the limit $J_{\mathrm{H}} \rightarrow \infty$ was introduced by Anderson and Hasegawa ${ }^{2}$ ) in the form

$$
\mathcal{H}=-\sum_{i j} t\left(\vec{S}_{i}, \vec{S}_{j}\right)\left(\tilde{c}_{i}^{\dagger} \tilde{c}_{j}+\text { h.c. }\right) .
$$

Mean-field type arguments of these models, including those by de Gennes, the magnetism of manganite compounds.

However, recent reinvestigations of these models which are motivated by the observation of colossal magnetoresistance (CMR) phenomenat in manganites revealed that such simplified treatments are insufficient to discuss quantitative nature of the models. Let us show an example. Curie temperature $T_{\mathrm{c}}$ of the model has been estimated by Millis et al 6 using the mean-field type discussion. Using some appropriate values for $t$ and $J_{\mathrm{H}}$, they found that the mean-field $T_{\mathrm{c}}$ of the model is much larger than those for perovskite manganites ( $\mathrm{La}, \mathrm{Sr}) \mathrm{MnO}_{3}$, and concluded that double-exchange alone is insufficient to explain the thermodynamics of these manganites. However, as we will show in this article, accurate treatments for the model give suppression of $T_{\mathrm{c}}$ from the mean-field value, and show consistency with $(\mathrm{La}, \mathrm{Sr}) \mathrm{MnO}_{3}$ data. 
Another important point which will be discussed here is the systematic understanding of experiments. For example, it is known that CMR manganites exhibit metal-insulator transition at Curie temperature. This statement is, however, inaccurate. By varying compositions, varieties of phases with different properties have been known.7. A typical reference compound $\mathrm{La}_{2} / 3 \mathrm{Sr}_{1 / 3} \mathrm{MnO}_{3}$ shows no metal-insulator transition. The resistivity always increases by the increase of temperature, i.e. $\mathrm{d} \rho(T) / \mathrm{d} T>0$ even above $T_{\mathrm{c}}$. Its absolute value in the paramagnetic phase is around the Mott's limit value, and may be explained by the DE model. On the other hand, $\mathrm{La}_{2 / 3} \mathrm{Ca}_{1 / 3} \mathrm{MnO}_{3}$ which is often reffered to as an "optimal" CMR material shows insulating behavior above $T_{\mathrm{c}}$ with much larger $\rho(T)$. Thus we have to be mindful to make distinctions between various compositions of manganites in a systematic manner.

The purpose of this article is to solve such misunderstandings and confusions in both theoretical and experimental studies. We review the investigation of the DE model by the author 6, 6, 10,11, 12, 13,14, 15, 16 The first part of this article shows the finite temperature behaviors in the DE systems. In $\S 2$ we introduce the model and the method (dynamical mean-field approach and the Monte Carlo calculation). In $\S 3$ the results for infinitely large spin (classical spin limit) is presented, and the $1 / S$ correction is shown in $\S 4$. The second part is devoted for discussions with respect to comparisons of model behavior with experimental data. Comparison with experiments. are shown in $\S 5$. Section 6 is devoted for concluding remarks.

\section{MODEL AND METHODS}

\subsection{Double Exchange Model in the Large Spin Limit}

Model. The compound $\mathrm{LaMnO}_{3}$ has four $3 \mathrm{~d}$ electrons per atom in the $\left(t_{2 \mathrm{~g}}\right)^{3}\left(e_{\mathrm{g}}\right)$ configuration. Due to Hund's coupling, these electrons have the high spin state, i.e. spin parallel configuration. By substitution of the La site with alkaline-earth divalent ions, holes are doped as carriers which is considered to enter the $e_{\mathrm{g}}$ orbitals.

As briefly mentioned in the previous section, Zener $\mathbb{B}$ introduced a Kondo-lattice type Hamiltonian (11) with ferromagnetic spin exchange $J_{\mathrm{H}}$ between localized spins and itinerant electrons. In manganites $(R, A) \mathrm{MnO}_{3}$, Hund's coupling $J_{\mathrm{H}}$ is estimated to be a few eV while electron hopping $t$ be in the order of $0.1 \mathrm{eV}$. Therefore, we have to deal with a strong coupling region $J_{\mathrm{H}} \gg t$. In order to discuss the ferromagnetism of the model in the metallic region, Zener introduced the notion of the "double exchange" interaction.

In some cases, the limit $J_{\mathrm{H}} \rightarrow \infty$ first studied by Anderson and Hasegawal is called the DE model. However, in order to avoid complications, we call the model (11) in the strong coupling region as DE model. In the weak coupling limit $J_{\mathrm{H}} / t \ll 1$, the model is often referred to as the $s-d$ model and studied as a model hamiltonian for the magnetic semiconductors.

The classical rotator limit, or equivalently large spin limit $S=\infty$, has been introduced by Anderson and Hasegawa.2 In manganites, we consider the case where the localized spin is in a high-spin state $(S=3 / 2)$ with the ferromagnetic coupling, the effect of quantum exchange seems to be less relevant compared to thermal fluctuations, at least in low energy physics. However, the role of quantum exchanges might give non-trivial effects in the system in the region with less thermal fluctuations. Such issues are left for future studies.

In this article, we introduce a model with finite $J_{\mathrm{H}}$ and infinite $S$,

$$
\mathcal{H}_{S=\infty}=-\sum_{i j, \sigma} t_{i j}\left(c_{i \sigma}^{\dagger} c_{j \sigma}+\text { h.c. }\right)-J_{\mathrm{H}} \sum_{i} \vec{\sigma}_{i} \cdot \vec{m}_{i}
$$

Hereafter we express the localized (classical) spin by $\vec{m}_{i}=\left(m_{i}{ }^{x}, m_{i}{ }^{y}, m_{i}{ }^{z}\right)$ with the normalization $|\vec{m}|^{2}=1$. 
Previous investigations. Anderson-Hasegawa 2 as well as de Gennes ${ }^{3}$ studied the magnetism of the model with localized spins treated as static, i.e. neglecting spin fluctuations, they made a search for energetically favored spin configurations as well as a mean-field calculation for finite temperatures. However, if we consider the system at finite temperature, especially around $T \sim T_{\mathrm{c}}$, spin fluctuations $\delta S_{i}$ become important. Especially, in our case of $J_{\mathrm{H}} \gg t$, such spin fluctuations give large effects to electronic structures since $J_{\mathrm{H}} \delta S \gg t$.

The point of interest for us in relationship with CMR phenomena is the change of resistivity $\rho$ at around $T_{\mathrm{c}}$. Mean-field type treatments are not justified for this purpose. An alternative approach has been made by Kubo and Ohata.17) They phenomenologically assumed the electron self-energy $\Sigma$ in the form

$$
\tau^{-1}=\operatorname{Im} \Sigma \propto 1-M^{2}
$$

where $\tau$ is the quasiparticle lifetime, and calculated the resistivity using the Drude's formula $\rho \propto \tau^{-1}$. Within this phenomenological treatment, the result qualitatively helps us to understand the magnetoresistance (MR) of the DE model via spin disorder scattering mechanism. However, in a quantitative way, it fails to reproduce the $\mathrm{MR}$ of $(\mathrm{La}, \mathrm{Sr}) \mathrm{MnO}_{3}$ as we will show in $\$ 5.1$.

Beyond the previous theories. Thus, in order to understand the behaviour of the DE model and to make direct comparisons with experimental data, it is very important to treat the model in an accurate way. The methods have to be an unbiased non-perturbative approaches since $J_{\mathrm{H}} \gg t$, and must take into account the effect of spin fluctuations to calculate finite temperature behaviors.

Here we introduce two methods: The dynamical mean-field (DFM) theory and the Monte Carlo calculation. Both of these methods give us the electronic states at finite temperature including $T \sim T_{\mathrm{c}}$. They are unbiased and become exact in the limit of large coordination number and large system size, respectively. One of the advantages of these methods, in the viewpoint of comparison with experiments, is that it is easy to obtain dynamical quantities such as density of states (DOS) and optical conductivity $\sigma(\omega)$.

\subsection{Dynamical mean-field theory}

We introduce the DMF method for the double-exchange model. For a general review of the field, see the review articles in refs. 18 and 19. Within the general scheme of the DMF, we treat a lattice system by considering a single-site coupled with "electron bath", or the time-dependent mean-field $\tilde{G}_{0}$. This method becomes exact in the large coordination number limit or equivalently large spatial dimension limit.20.21)

Generic part of the DMF treatment is as follows. Solving a model-specific single-site problem, we obtain the self-energy $\tilde{\Sigma}\left(\mathrm{i} \omega_{n}\right)$ from $\tilde{G}_{0}$. Lattice Green's function is approximated by

$$
G\left(k, \mathrm{i} \omega_{n}\right)=\left[\mathrm{i} \omega_{n}-(\varepsilon-\mu)-\tilde{\Sigma}\left(\mathrm{i} \omega_{n}\right)\right]^{-1} .
$$

The local Green's function is defined by

$$
G_{\text {loc }}\left(\mathrm{i} \omega_{n}\right) \equiv \frac{1}{N} \sum_{k} G\left(k, \mathrm{i} \omega_{n}\right)
$$

Since $\tilde{\Sigma}$ is $k$-independent, $k$ dependence of $G\left(k, i \omega_{n}\right)$ comes through the energy dispersion and we have

$$
G_{\text {loc }}\left(\mathrm{i} \omega_{n}\right)=\int \mathrm{d} \varepsilon N_{0}(\varepsilon)\left[\mathrm{i} \omega_{n}-(\varepsilon-\mu)-\tilde{\Sigma}\left(\mathrm{i} \omega_{n}\right)\right]^{-1} .
$$

$N_{0}(\varepsilon)$ is the DOS for the noninteracting lattice system. The information of the lattice geometry is included through the noninteracting DOS in eq. (7). The method is applicable to finite size 
systems by taking the sum over $k$ points in eq. (6) in the discrete $k$-space. We self-consistently obtain the time-dependent mean-field $\tilde{G}_{0}$ as

$$
\tilde{G}_{0}\left(\mathrm{i} \omega_{n}\right)=\left(G_{\text {loc }}^{-1}\left(\mathrm{i} \omega_{n}\right)+\tilde{\Sigma}\left(\mathrm{i} \omega_{n}\right)\right)^{-1} .
$$

Let us now discuss the model-specific part.8) For the present system (3), the action of the effective single-site model is described as

$$
\begin{aligned}
\tilde{S}= & -\int_{0}^{\beta} \mathrm{d} \tau_{1} \int_{0}^{\beta} \mathrm{d} \tau_{2} \Psi^{*}\left(\tau_{1}\right) \tilde{G}_{0}^{-1}\left(\tau_{1}-\tau_{2}\right) \Psi\left(\tau_{2}\right) \\
& -J_{\mathrm{H}} \int_{0}^{\beta} \mathrm{d} \tau \vec{m} \cdot \Psi^{*}(\tau) \vec{\sigma} \Psi(\tau) .
\end{aligned}
$$

Here $\Psi^{*}=\left(c_{\uparrow}^{*}, c_{\downarrow}^{*}\right)$ is the Grassmann variables in the spinor notation. Green's function in the imaginary time is calculated as

$$
\begin{aligned}
\tilde{G}\left(\mathrm{i} \omega_{n}\right) & =\left\langle\left(\tilde{G}_{0}^{-1}\left(\mathrm{i} \omega_{n}\right)+J_{\mathrm{H}} \vec{m} \cdot \vec{\sigma}\right)^{-1}\right\rangle \\
& =\frac{1}{\tilde{Z}} \int \mathrm{d} \Omega P(\vec{m})\left(\tilde{G}_{0}^{-1}\left(\mathrm{i} \omega_{n}\right)+J_{\mathrm{H}} \vec{m} \cdot \vec{\sigma}\right)^{-1} .
\end{aligned}
$$

$P(\vec{m})$ is the Boltzmann factor for the spin

$$
P(\vec{m})=\frac{1}{\tilde{Z}} \exp \left[-\tilde{S}_{\text {eff }}(\vec{m})\right]
$$

where $\tilde{S}_{\text {eff }}$ is the effective action for the spin

$$
\begin{aligned}
& \tilde{S}_{\text {eff }}(\vec{m})=-\log \operatorname{Tr}_{\mathrm{F}} \exp (-\tilde{S}) \\
& =-\sum_{n} \log \operatorname{det}\left[\frac{1}{\mathrm{i} \omega_{n}}\left(\tilde{G}_{0}^{-1}\left(\mathrm{i} \omega_{n}\right)+J_{\mathrm{H}} \vec{m} \vec{\sigma}\right)\right] \mathrm{e}^{\mathrm{i} \omega_{n} 0_{+}} .
\end{aligned}
$$

$\tilde{Z}$ is the partition function

$$
\begin{aligned}
\tilde{Z} & =\int \mathrm{d} \Omega_{\vec{m}} \int \mathcal{D} \Psi^{*} \mathcal{D} \Psi \exp (-\tilde{S}) \\
& =\int \mathrm{d} \Omega_{\vec{m}} \exp \left[-\tilde{S}_{\text {eff }}(\vec{m})\right] .
\end{aligned}
$$

The self energy for the single-site system $\tilde{\Sigma}$ is obtained from $\tilde{\Sigma}\left(\mathrm{i} \omega_{n}\right)=\tilde{G}_{0}^{-1}\left(\mathrm{i} \omega_{n}\right)-\tilde{G}^{-1}\left(\mathrm{i} \omega_{n}\right)$.

Magnetization of the local spin is obtained by

$$
\langle\vec{m}\rangle=\int \mathrm{d} \Omega_{\vec{m}} P(\vec{m}) \vec{m} .
$$

Hereafter we take the axis of the magnetization in $z$ direction and the order parameter is expressed as $M=\left\langle m_{z}\right\rangle$. Transport properties are obtained through the Kubo formula. Conductivity in $D=\infty$ is calculated as $22,23,24)$

$$
\sigma(\omega)=\sigma_{0} \sum_{\sigma} \int \mathrm{d} \omega^{\prime} I_{\sigma}\left(\omega^{\prime}, \omega^{\prime}+\omega\right) \frac{f\left(\omega^{\prime}\right)-f\left(\omega^{\prime}+\omega\right)}{\omega},
$$

where

$$
I_{\sigma}\left(\omega_{1}, \omega_{2}\right)=\int N_{0}(\epsilon) \mathrm{d} \epsilon W^{2} A_{\sigma}\left(\epsilon, \omega_{1}\right) A_{\sigma}\left(\epsilon, \omega_{2}\right)
$$


Here, the spectral weight function is defined by

$$
A_{\sigma}(\epsilon, \omega)=-\frac{1}{\pi} \operatorname{Im} G_{\sigma}(\epsilon, \omega+\mathrm{i} \eta)
$$

while $f$ is the Fermi distribution function. The constant $\sigma_{0}$ gives the unit of conductivity. In this formula, we used that the vertex correction cancels in the conductivity calculation at infinitedimensional limit.25) Thermopower is also calculated: The Seebeck coefficient is obtained as 26, 18)

$$
S=\frac{1}{e T} \frac{L_{2}}{L_{1}}
$$

where $L_{k}(k=1,2)$ is defined by

$$
L_{k}=\sum_{\sigma} \int \mathrm{d} \omega\left(-\frac{\partial f(\omega)}{\partial \omega}\right) I_{\sigma}(\omega, \omega)(\beta \omega)^{k-1} .
$$

The method is easily expanded to a Bethe lattice with two-sublattice symmetry. In this case, magnetic phases with ferromagnetic and antiferromagnetic order parameters can be considered simultaneously. We introduce $\alpha=A, B$ sublattice indices for the Weiss fields $\tilde{G}_{0 \alpha}\left(\mathrm{i} \omega_{n}\right)$, and solve coupled self-consistency equations. The formula to calculate the Green's function is now given by

$$
\tilde{G}_{\alpha}\left(\mathrm{i} \omega_{n}\right)=\int \mathrm{d} \Omega_{\alpha} P_{\alpha}(\vec{m})\left(\tilde{G}_{0 \alpha}^{-1}\left(\mathrm{i} \omega_{n}\right)+J \vec{m} \cdot \vec{\sigma}\right)^{-1} .
$$

Here, the Boltzmann weight for the configuration of local spin $P_{\alpha}(\vec{m})$ is calculated from the effective action $\tilde{S}_{\alpha}$,

$$
\begin{aligned}
\tilde{S}_{\alpha}(\vec{m}) & =-\log \operatorname{Tr}_{\mathrm{F}} \exp \left[-\tilde{S}\left(\tilde{G}_{0 \alpha}, \vec{m}\right)\right] \\
P_{\alpha}(\vec{m}) & =\exp \left[-\tilde{S}_{\alpha}(\vec{m})\right] / \tilde{Z}_{\alpha}, \\
\tilde{Z}_{\alpha} & =\int \mathrm{d} \Omega_{\alpha} \exp \left[-\tilde{S}_{\alpha}(\vec{m})\right]
\end{aligned}
$$

Integration over DOS as in eq. (8) gives the self-consistent mapping relation 27]

$$
\begin{aligned}
& \tilde{G}_{0 A}{ }^{-1}\left(\mathrm{i} \omega_{n}\right)=\mathrm{i} \omega_{n}+\mu-\tilde{G}_{B}\left(\mathrm{i} \omega_{n}\right) W^{2} / 4, \\
& \tilde{G}_{0 B}{ }^{-1}\left(\mathrm{i} \omega_{n}\right)=\mathrm{i} \omega_{n}+\mu-\tilde{G}_{A}\left(\mathrm{i} \omega_{n}\right) W^{2} / 4 .
\end{aligned}
$$

Now the self-consistency equations (20)-(24) form a closed set. Within this approach, we can study the instability and the formation of magnetic ordering with ferromagnetic, antiferromagnetic, and canted antiferromagnetic symmetries.

\subsection{Analytical solutions of the dynamical mean-field theory}

In some limiting cases, analytical solutions are available for the DMF calculations. Analytical expressions are in general quite useful to obtain intuitions.

Paramagnetic phase. For the paramagnetic solution, Green's function is a scalar function with respect to spin rotation, so we have $\tilde{G}_{0}\left(\mathrm{i} \omega_{n}\right)=\tilde{g}_{0}\left(\mathrm{i} \omega_{n}\right) I$ where $I$ is the $2 \times 2$ eigenmatrix. Then, we have

$$
\tilde{G}\left(\mathrm{i} \omega_{n}\right)=\left\langle\frac{\tilde{g}_{0}\left(\mathrm{i} \omega_{n}\right)^{-1} I-J_{\mathrm{H}} \vec{m} \vec{\sigma}}{\tilde{g}_{0}\left(\mathrm{i} \omega_{n}\right)^{-2}-J_{\mathrm{H}}^{2}|\vec{m}|^{2}}\right\rangle .
$$


Since $\langle\vec{m}\rangle=0$ and $\left\langle|\vec{m}|^{2}\right\rangle=1$, we have

$$
\tilde{G}\left(\mathrm{i} \omega_{n}\right)=\frac{\tilde{g}_{0}\left(\mathrm{i} \omega_{n}\right)^{-1}}{\tilde{g}_{0}\left(\mathrm{i} \omega_{n}\right)^{-2}-J_{\mathrm{H}}^{2}} I=\frac{1}{2}\left(\frac{1}{\tilde{g}_{0}\left(\mathrm{i} \omega_{n}\right)^{-1}+J_{\mathrm{H}}}+\frac{1}{\tilde{g}_{0}\left(\mathrm{i} \omega_{n}\right)^{-1}+J_{\mathrm{H}}}\right) I .
$$

The self-energy is then given by

$$
\Sigma\left(\mathrm{i} \omega_{n}\right)=\tilde{G}_{0}^{-1}\left(\mathrm{i} \omega_{n}\right)-\tilde{G}^{-1}\left(\mathrm{i} \omega_{n}\right)=J_{\mathrm{H}}^{2} \tilde{G}_{0}\left(\mathrm{i} \omega_{n}\right) .
$$

From the derivation, we see that the Green's function (26) is the same as that of the system with the Ising substrate spin $\vec{m}=(0,0, \pm 1)$. Furthermore, the Green's function (26) shares the same analytical structure as that of the infinite-dimensional Falicov-Kimball model (FKM) (or, simplified Hubbard model)

$$
\mathcal{H}_{\mathrm{FKM}}=-\sum_{i j} t_{i j}\left(c_{i}^{\dagger} c_{j}+\text { h.c. }\right)+U \sum_{i} c_{i}^{\dagger} c_{i} f_{i}^{\dagger} f_{i}
$$

at $\left\langle n_{\mathrm{f}}\right\rangle=1 / 2$. Green's function of the FKM in infinite dimension is described as 22, 29)

$$
\tilde{G}_{\mathrm{FKM}}\left(\mathrm{i} \omega_{n}\right)=\frac{1-\left\langle n_{\mathrm{f}}\right\rangle}{\tilde{G}_{0}^{-1}\left(\mathrm{i} \omega_{n}\right)}+\frac{\left\langle n_{\mathrm{f}}\right\rangle}{\tilde{G}_{0}^{-1}\left(\mathrm{i} \omega_{n}\right)-U} .
$$

Then, the Green's functions (26) and (29) share the same analytical structure at $\left\langle n_{\mathrm{f}}\right\rangle=1 / 2$. In the FKM, the c-electrons are scattered by the charge fluctuations of the localized f-electrons, which corresponds to the scattering process of the itinerant electrons by the localized spins in the DE model.

Hence, thermodynamical properties of the DE model in $D=\infty$ and $S=\infty$ can be understood from the nature of the FKM in $D=\infty$ which has been studied intensively. For example, the imaginary part of the self-energy at the fermi level is finite $\operatorname{Im} \Sigma(0) \neq 0$ in the paramagnetic phase.22, 29)

In Fig. 1(a) we schematically illustrate the spectral function $A(k, \omega)$ in the paramagnetic phase. As in the case for FKM, the spectral weight is split into two parts at $\omega \sim \pm J_{\mathrm{H}}$ for sufficiently large Hund's coupling $J_{\mathrm{H}} \gg W$. For the semi-circular density of states with the bandwidth $W$, we have the metal-insulator transition 30 at $J_{\mathrm{H}}{ }^{\mathrm{c}}=0.5 \mathrm{~W}$, which has the Hubbard-III like nature. Kondo resonance peak, which is seen in the Hubbard model31, 32.33) is missing in this model, since the quantum exchange process is absent. As the magnetic moment is induced, the imaginary part of the self-energy decreases because the thermal fluctuation of spins decreases. At the ground state, spins are magnetically ordered. For the ferromagnetic ground state, there exists two free electron bands which are energetically split by $J_{\mathrm{H}}$ exchange interactions.

Infinite $J_{\mathrm{H}}$ limit -Green's function-. In the case of Lorentzian DOS, Green's function is easily obtained in the limit $J_{\mathrm{H}} \rightarrow \infty$.10) We consider the hole doped region where $\mu \sim-J_{\mathrm{H}}$. In the case of the Lorentzian DOS, the self-consistency equation gives

$$
G_{0}(\omega+\mathrm{i} \eta)=\left(\Omega-J_{\mathrm{H}}+\mathrm{i} W\right)^{-1} .
$$

Here, chemical potential is $\mu=-J_{\mathrm{H}}+\delta \mu$ where $\delta \mu=O(W)$, and $\Omega \equiv \omega+\delta \mu=O(W)$ is the energy which is measured from the center of the lower sub-band $-J_{\mathrm{H}}$.

Magnetic field in the $z$ direction is applied to the localized spins in the paramagnetic phase, and the induced magnetization is expressed as $M=\left\langle m_{z}\right\rangle$. Since $G_{0}$ in eq. (30) is spin independent even in the spin polarized cases, eqs. (25) and (30) gives

$$
\begin{aligned}
G_{\sigma}(\omega+\mathrm{i} \eta)= & \frac{\left(\Omega-J_{\mathrm{H}}+\mathrm{i} W\right)-J_{\mathrm{H}} M \sigma}{\left(\Omega-J_{\mathrm{H}}+\mathrm{i} W\right)^{2}-J_{\mathrm{H}}{ }^{2}} \\
= & \frac{1+M \sigma}{2} \frac{1}{\Omega+\mathrm{i} W}+O\left(1 / J_{\mathrm{H}}\right) . \\
& -6-
\end{aligned}
$$




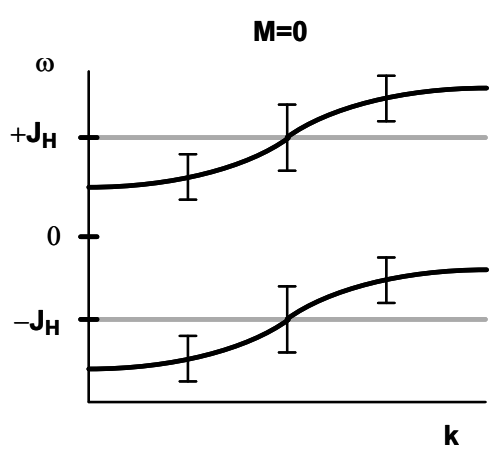

(a)

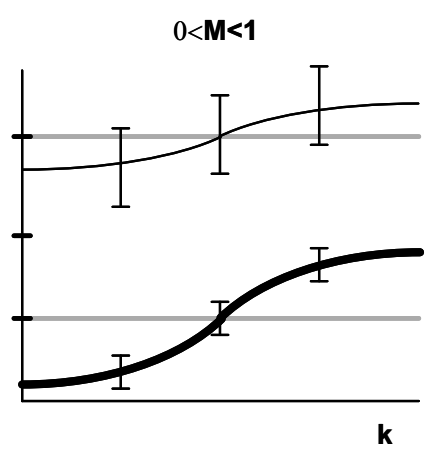

(b)

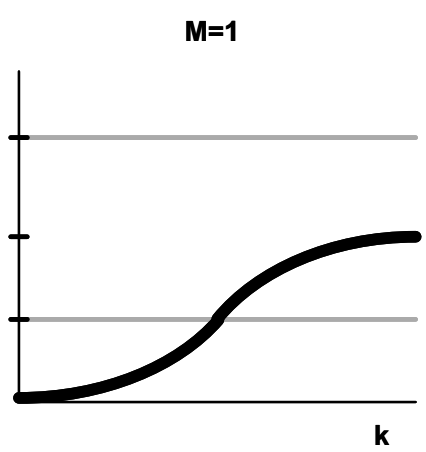

(c)

Figure 1: Schematic behavior in the spectral function for up-spin electrons, at (a) paramagnetic state $M=0$, (b) ferromagnetic state at $0<T<T_{\mathrm{c}}$, and (c) at the ground state $T=0$ and $M=1$. Solid curves illustrate peak positions of $A(k, \omega)$. Width of the curves represent height of the peak (quasiparticle weight), while error bars represent the linewidth (inverse of lifetime). Grey lines are guides to eyes. Spectral functions for down-spin electrons are obtained by exchanging upper part $\left(\omega \sim J_{\mathrm{H}}\right)$ and lower part $\left(\omega \sim-J_{\mathrm{H}}\right)$. Also see Fig. 4 in $\S 3.1$ for actual data.

At $J_{\mathrm{H}} / W \rightarrow \infty$, the spectral weight is calculated as

$$
A_{\sigma}(\omega)=-\frac{1}{\pi} \mathcal{I m} G_{\sigma}(\omega+\mathrm{i} \eta)=\frac{1+M \sigma}{2} \cdot \frac{1}{\pi} \frac{W}{\Omega^{2}+W^{2}} .
$$

We see that the center of the spectral weight is indeed shifted to $-J_{\mathrm{H}}$. The amplitude of $A_{\sigma}$ is proportional to the population of the local spins parallel to $\sigma$, which indicates that the electronic states that are anti-parallel to the local spin are projected out. The self-energy is calculated from eqs. (30) and (31) as

$$
\Sigma_{\sigma}(\omega+\mathrm{i} \eta)=-J_{\mathrm{H}}-\frac{1-M \sigma}{1+M \sigma}(\Omega+\mathrm{i} W) .
$$

Eq. (33) gives $\operatorname{Re} \Sigma \sim-J_{\mathrm{H}}$, so the shift in $\mu$ is self-consistently justified again.

Similarly, Green's function at $\omega^{\prime} \sim 2 J_{\mathrm{H}}$, namely at the upper subband, is described as follows. Using $\omega^{\prime}=\omega+2 J_{\mathrm{H}}$, where $\omega=O(W)$, Green's function is given by

$$
G_{\sigma}\left(\omega+2 J_{\mathrm{H}}+\mathrm{i} \eta\right)=\frac{1-M \sigma}{2} \frac{1}{\Omega+\mathrm{i} W}+O\left(1 / J_{\mathrm{H}}\right),
$$

and the spectral weight is calculated as

$$
A_{\sigma}\left(\omega+2 J_{\mathrm{H}}\right)=\frac{1-M \sigma}{2} \cdot \frac{1}{\pi} \frac{W}{\Omega^{2}+W^{2}} .
$$

From eqs. (32) and (35), we see the transfer of the spectral weight by magnetization.

From above equations, Green's function is given in the form

$$
\begin{aligned}
& G_{\sigma}(k, \omega ; M)= \frac{z_{\sigma}^{(1)}(M)}{\omega+J_{\mathrm{H}}+\mu-\zeta_{\sigma}^{(1)}(k ; M)+\mathrm{i} \Gamma_{\sigma}^{(1)}(M)} \\
&+\frac{z_{\sigma}^{(\mathrm{u})}(M)}{\omega-J_{\mathrm{H}}+\mu-\zeta_{\sigma}^{(\mathrm{u})}(k ; M)+\mathrm{i} \Gamma_{\sigma}^{(\mathrm{u})}(M)}, \\
&-7-
\end{aligned}
$$


asymptotically at $J_{\mathrm{H}} / W \rightarrow \infty$. In this limit, Green's function is a sum of contributions from lower subbandrs (l) and upper subband (u). Quasiparticle residue is given by

$$
z_{\sigma}^{(\mathrm{l})}(M)=P_{\sigma}^{+}(M), \quad z_{\sigma}^{(\mathrm{u})}(M)=P_{\sigma}^{-}(M),
$$

quasiparticle dispersion relation is described as

$$
\zeta_{\sigma}^{(1)}(k ; M)=P_{\sigma}^{+}(M) \varepsilon_{k}, \quad \zeta_{\sigma}^{(\mathrm{u})}(k ; M)=P_{\sigma}^{-}(M) \varepsilon_{k},
$$

and the quasiparticle linewidth is in the form

$$
\Gamma_{\sigma}^{(\mathrm{l})}(M)=P_{\sigma}^{-}(M) W, \quad \Gamma_{\sigma}^{(\mathrm{u})}(M)=P_{\sigma}^{+}(M) W .
$$

Here, $P$ are the function of spin polarization

$$
P_{\sigma}^{+}(M) \equiv \frac{1+M \sigma}{2}, \quad P_{\sigma}^{-}(M) \equiv \frac{1-M \sigma}{2} .
$$

In Fig. 1 we schematically show the spectral function for the up-spin electron $A_{\uparrow}(k, \omega)=$ $-\operatorname{Im} G_{\uparrow}(k, \omega) / \pi$ calculated from eq. (36). For the down-spin electrons, $A_{\downarrow}(k, \omega)$ is obtained by replacing upper and lower subbands, e.g. $z_{\downarrow}^{(1)}=z_{\uparrow}^{(\mathrm{u})}$, etc. In the paramagnetic phase (Fig. 11(a)), quasiparticle dispersion are split into lower and upper subbands at $\omega \sim \pm J_{\mathrm{H}}$ with quasiparticle weight $z_{\uparrow}^{(\mathrm{l})}=z_{\uparrow}^{(\mathrm{u})}=1 / 2$. As magnetization is increased below $T_{\mathrm{c}}$, the lower subband gains quasiparticle weight $z_{\uparrow}^{(1)}=(1+M) / 2$ and that for upper subband decreases as $z_{\uparrow}^{(\mathrm{u})}=(1-M) / 2$. At the ground state with perfect spin polarization $M=1$, the electronic Hamiltonian describes a free electron system under Zeeman splitting field. Hence there exists only lower subband for the up spin electron. This limit is described as $z_{\uparrow}^{(\mathrm{l})}=1$ and $z_{\uparrow}^{(\mathrm{u})}=0$.

In the paramagnetic phase $M=0$ we see $\operatorname{Im} \Sigma=-W$, which means that the quasi-particle excitation is very incoherent; the lifetime of a quasi-particle is comparable with the time scale that an electron transfers from site to site. This result justifies us to take the $D=\infty$ limit which is essentially a single-site treatment.

In the following section ( $\S 3.1)$, we will discuss the behavior of the spectral function for general cases.

Infinite $J_{\mathrm{H}}$ limit -Curie temperature-- In the semicircular DOS case, we consider the ferromagnetic state under doping at $J_{\mathrm{H}} \gg W$. We set

$$
G_{0}^{-1}\left(\mathrm{i} \omega_{n}\right)=\left(\mathrm{i} \omega_{n}+R_{n}\right) \hat{I}+Q_{n} \hat{\sigma}_{z}
$$

and $\mu=-J_{\mathrm{H}}+\delta \mu$. In order to keep the carrier concentration finite, we take the limit $J_{\mathrm{H}} \rightarrow \infty$ with keeping $\delta \mu=O(W)$. In this limit, we have

$$
R_{n}=-\frac{W^{2}}{8}\left\langle\frac{1}{z_{n}+R_{n}+m_{z} Q_{n}}\right\rangle, \quad Q_{n}=-\frac{W^{2}}{8}\left\langle\frac{m_{z}}{z_{n}+R_{n}+m_{z} Q_{n}}\right\rangle,
$$

where $z_{n}=\mathrm{i} \omega_{n}+\delta \mu$. The Boltzmann weight is calculated from eq. (12) as

$$
P(\vec{m}) \propto \exp \left[\sum_{n} \log \left(1+\frac{R_{n}+Q_{n} m_{z}}{z_{n}}\right)\right] .
$$

This equation tells us that the model is not simply mapped to the Heisenberg model. The Boltzmann weight of the Heisenberg model in infinite dimension is expressed as $P_{\text {Heis }}(\vec{m}) \sim$ $\exp \left(-\beta h_{\mathrm{eff}} m_{z}\right)$, which contradicts with that of the DE model, i.e. $P(\vec{m}) \neq P_{\text {Heis }}$. This reflects 


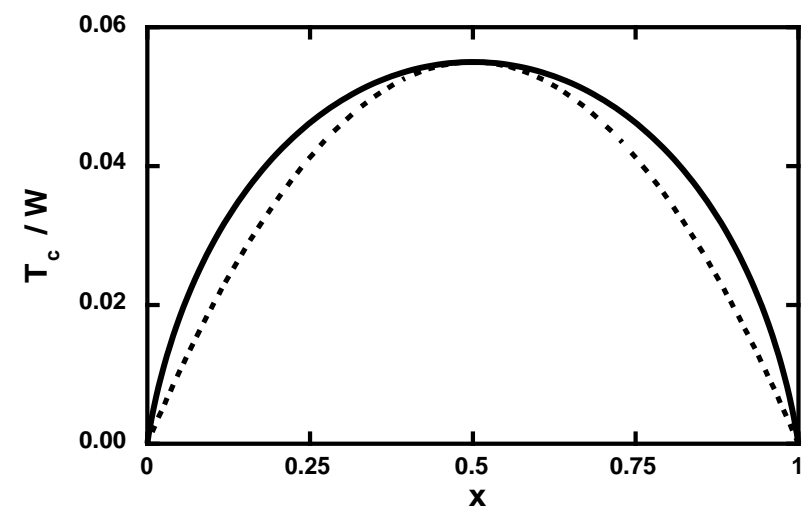

Figure 2: Curie temperature for the semicircular DOS at $J_{\mathrm{H}}=\infty$ (solid curve). Dotted curve is the form $T_{\mathrm{c}} \propto x(1-x)$.

the fact that the itinerant ferromagnet has smaller $T_{\mathrm{c}}$ due to the spin fluctuation affecting the itinerant electrons, compared to the insulating ferromagnet with the same spin stiffness.

Let us calculate $T_{\mathrm{c}}$. At $T \sim T_{\mathrm{c}}$, we have $M=\langle m\rangle \ll 1$ and

$$
\begin{aligned}
R_{n} & =-\frac{W^{2}}{8} \frac{1}{z_{n}+R_{n}}+O(M) \\
Q_{n} & =-\frac{W^{2}}{8}\left\langle\frac{m_{z}}{z_{n}+R_{n}}-\frac{m_{z}^{2}}{\left(z_{n}+R_{n}\right)^{2}} Q_{n}\right\rangle+O\left(M^{2}\right) .
\end{aligned}
$$

By solving eqs. (44) and (45), we have

$$
R_{n}=\sqrt{z_{n}{ }^{2}-W^{2} / 4}-z_{n}, \quad \frac{Q_{n}}{M}=\frac{R_{n}}{1-8\left\langle m_{z}{ }^{2}\right\rangle R_{n}^{2} / W^{2}} .
$$

Here, $\left\langle m_{z}{ }^{2}\right\rangle \equiv \int \mathrm{d} \Omega m_{z}{ }^{2}=1 / 3$ at $S=\infty$. The Boltzmann weight is given by

$$
P(\vec{m}) \propto \exp \left[\sum_{n} \frac{Q_{n}}{z_{n}+R_{n}} m_{z}\right]=\exp \left(-\beta J_{\text {eff }} M m_{z}\right)
$$

where

$$
J_{\mathrm{eff}}(\beta)=\frac{1}{\beta} \sum_{n} \frac{8 R_{n}^{2} / W^{2}}{1-8 R_{n}^{2} /\left(3 W^{2}\right)} .
$$

Then, the partition function is identical to that of the Heisenberg model with exchange coupling $J_{\text {eff }}$, and $T_{\mathrm{c}}$ is obtained from

$$
T_{\mathrm{c}}=\left.\frac{1}{\beta} \sum_{n} \frac{8 R_{n}^{2}}{3 W^{2}-8 R_{n}^{2}}\right|_{\beta=1 / T_{\mathrm{c}}} .
$$

Solving eqs. (46) and (49) self-consistently, we obtain $T_{\mathrm{c}}$ as a function of $\mu$, while the carrier number is calculated directly from Green's function $G\left(\mathrm{i} \omega_{n}\right)$. In Fig. 2 we plot $T_{\mathrm{c}}$ as a function of doping $x$. We see that $T_{\mathrm{c}}$ has a maximum at $x=0.5$ with $T_{\mathrm{c}} \sim 0.05 W$. Then, using $\beta W \ll 1$ we may approximate the summation in eq. (49) by integration,

$$
T_{\mathrm{c}}=\frac{1}{2 \pi} \int_{-\infty}^{\infty} \mathrm{d} x \frac{8 R^{2}(\mathrm{i} x+\delta \mu)}{3 W^{2}-8 R^{2}(\mathrm{i} x+\delta \mu)}
$$

where $R(z)=\sqrt{z^{2}-W^{2} / 4}-z$. 
The result is particle-hole symmetric, namely $T_{\mathrm{c}}(x)=T_{\mathrm{c}}(1-x)$. At $x \rightarrow 0$ and $x \rightarrow 1, T_{\mathrm{c}}$ diminishes because the ferromagnetism of this system is due to the kinetic energy of the conduction electron. In Fig. 2, we also depict a curve $T_{\mathrm{c}} \propto x(1-x)$ proposed in ref. 34 for comparison. This simple form roughly reproduces the doping dependence of $T_{\mathrm{c}}$. In the following section, we discuss more precisely about the Curie temperature at finite $J_{\mathrm{H}} / W$.

\subsection{Monte Carlo method for finite size clusters}

On a finite-size clusters, it is possible to investigate the double-exchange system by numerical methods. The result is unbiased and exact within the numerical errors.

The partition function of the present model with localized spins treated as classical rotators is defined by

$$
Z=\operatorname{Tr}_{\mathrm{S}} \operatorname{Tr}_{\mathrm{F}} \exp \left(-\beta\left[\mathcal{H}\left(\left\{\vec{m}_{i}\right\}\right)-\mu \hat{N}\right]\right)
$$

where $\operatorname{Tr}_{\mathrm{S}}$ and $\operatorname{Tr}_{\mathrm{F}}$ represent traces over spin and fermion degrees of freedom, respectively. In the finite size system, $Z$ is obtained by taking the trace over fermion degrees of freedom first and spin degrees of freedom afterwards. Fermion trace is directly calculated from the diagonalization of $2 N \times 2 N$ Hamiltonian matrix, where $N$ is the number of sites. Trace over spin degrees of freedom is replaced by the Monte Carlo summation over spin configurations $\left\{\vec{m}_{i}\right\}$.

For a fixed configuration of classical spins $\left\{\vec{m}_{i}\right\}$, the Hamiltonian is numerically diagonalized and we obtain

$$
\operatorname{Tr}_{\mathrm{F}} \exp \left(-\beta\left[\mathcal{H}\left(\left\{\vec{m}_{i}\right\}\right)-\mu N\right]\right)=\prod_{\nu=1}^{2 N}\left[1+\exp \left(-\beta\left(E_{\nu}\left(\left\{\vec{m}_{i}\right\}\right)-\mu\right)\right)\right]
$$

$E_{\nu}(\nu=1, \ldots, 2 N)$ are eigenvalues of the Hamiltonian matrix for a given configuration $\left\{\vec{m}_{i}\right\}$. We have the effective action for the classical spin system

$$
S_{\text {eff }}\left(\left\{\vec{m}_{i}\right\}\right)=-\sum_{\nu} \log \left(1+\mathrm{e}^{-\beta\left(E_{\nu}-\mu\right)}\right)
$$

which gives

$$
Z=\operatorname{Tr}_{\mathrm{S}} \exp \left(-S_{\mathrm{eff}}\right)
$$

Monte Carlo update of spin configurations is performed using the Boltzmann weight of the state $\left\{\vec{m}_{i}\right\}$

$$
P\left(\left\{\vec{m}_{i}\right\}\right) \propto \exp \left(-S_{\text {eff }}\left(\left\{\vec{m}_{i}\right\}\right)\right) .
$$

In a Monte Carlo unit step, orientations of each spins are updated using the Metropolis algorithm. Since the spins are classical, spin updates can be performed ergodically.

Thermodynamic quantities are stochastically calculated. Quantities which are associated with localized spins are obtained directly from the thermal average of spin configurations. Electronic quantities are calculated from the eigenvalues and eigenfunctions of $\mathcal{H}\left(\left\{\vec{m}_{i}\right\}\right)$.

One of the advantages for taking the classical spin limit is that there exists no "negative sign problem", which is present in quantum spin models. In the classical spin limit, the spin degrees of freedom is completely decoupled from those of fermions, and the fermionic trace in eq. (52) is obtained by solving the noninteracting lattice fermion system with random static potential. Another advantage is that the real frequency dynamics of electronic properties are directly obtained, since eigenvalues of the Hamiltonian are calculated through the Monte Carlo procedures. There is no difficulties of analytical continuations form imaginary frequencies, as is present in some quantum Monte Carlo methods. A disadvantage of this method is that the auxiliary field is static i.e. non-local in imaginary time, so it is not possible to make lacal spin flip using the imaginary time Green's function as in the case for the Hubbard model.35, 36 ) 
(a)

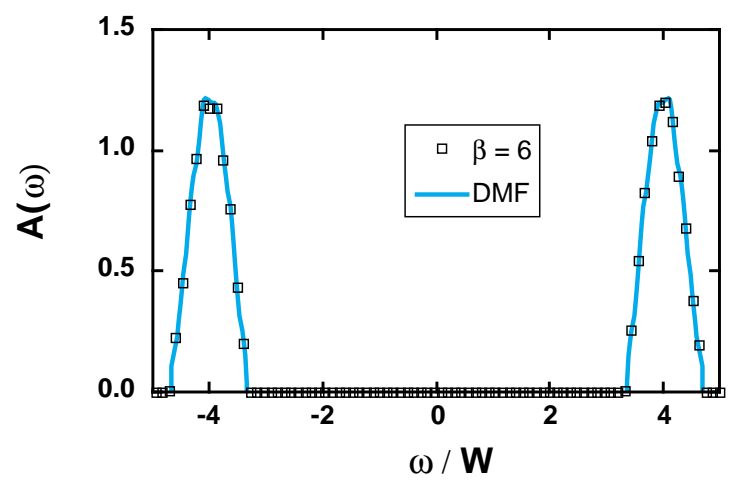

(b)

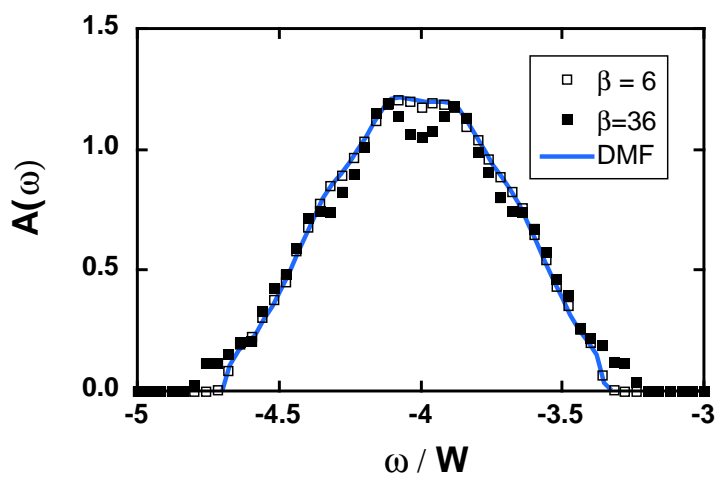

Figure 3: Monte Carlo results for DOS on $6 \times 4 \times 4$ cubic lattice at $J_{\mathrm{H}} / W=4$, at $\beta \equiv$ $W / T=6$ and 36 . Error bars are within the symbol size. Curves in the figure show the DMF results. (left) Two peak structure at $\omega \sim \pm J_{\mathrm{H}}$ is seen for both DMF and Monte Carlo results. (right) Lower subband at $\omega \sim-J_{\mathrm{H}}$.

Let us now compare the dynamical mean-field theory and the cluster Monte Carlo method. Here we calculate the electron DOS

$$
A(\omega)=-\frac{1}{N} \sum_{k \sigma} \operatorname{Im} G_{\sigma}(k, \omega+\mathrm{i} \eta) / \pi
$$

on a finite size cluster system in Fig. 3. We treat $N=6 \times 4 \times 4$ cubic lattice at $J_{\mathrm{H}} / W=4$ and $\mu=-J_{\mathrm{H}}$, where the dynamical mean-field approach gives $T_{\mathrm{c}}=0.028 \mathrm{~W}$. In order to avoid delta-function singularities in finite size systems, we use an adiabatic factor $\eta=10^{-2}$ to smooth the spectra. In the Monte Carlo calculation, we calculate at $\beta \equiv W / T=6$ and 36 , and compare with the dynamical mean-field result in the paramagnetic phase $T>T_{\mathrm{c}}$. The result shows that the DMF calculation is very accurate.

\section{RESULTS}

In this section, we show the results for the classical spin limit $S=\infty$ of the DE system using the DMF approach as well as the Monte Carlo calculation. Hereafter, the electronic bandwidth is taken to be $W \equiv 1$ as a unit of energy. For the carrier electron number, we express by $x=1-\langle n\rangle$. We define $M=\left\langle m_{z}\right\rangle$, where $0 \leq M \leq 1$. Also, we describe the total moment, or sum of moments of localized spin and electron spin, as $M_{\mathrm{tot}}=\left\langle\frac{3}{2} m_{z}+\frac{1}{2} \sigma_{z}\right\rangle$. In a normalized form we describe $M^{*}=M_{\text {tot }} / M_{\text {sat }}$, where $M_{\text {sat }}$ is the saturation value of $M_{\text {tot }}$ at the ground state. We make distinctions between $M$ and $M^{*}$, since analytical calculation is better understood by $M$ while the comparison with experiments should be done by $M^{*}$. Nevertheless, in the strong coupling region $J_{\mathrm{H}} \gg W$ we have $M \simeq M^{*}$ so effectively there exists no major differences.

\subsection{Electronic structures}

Spectral function and the density of states. In Fig. 4 we show the spectral function for the up-spin electron $A_{\uparrow}(k, \omega)$ on a cubic lattice, where $k / \pi=(\zeta, \zeta, \zeta)$. From the particle-hole symmetry and the spin symmetry, down-spin part $A_{\downarrow}(k, \omega)$ is reproduced by the relation

$$
A_{\downarrow}(k, \omega)=A_{\uparrow}(Q-k,-\omega),
$$



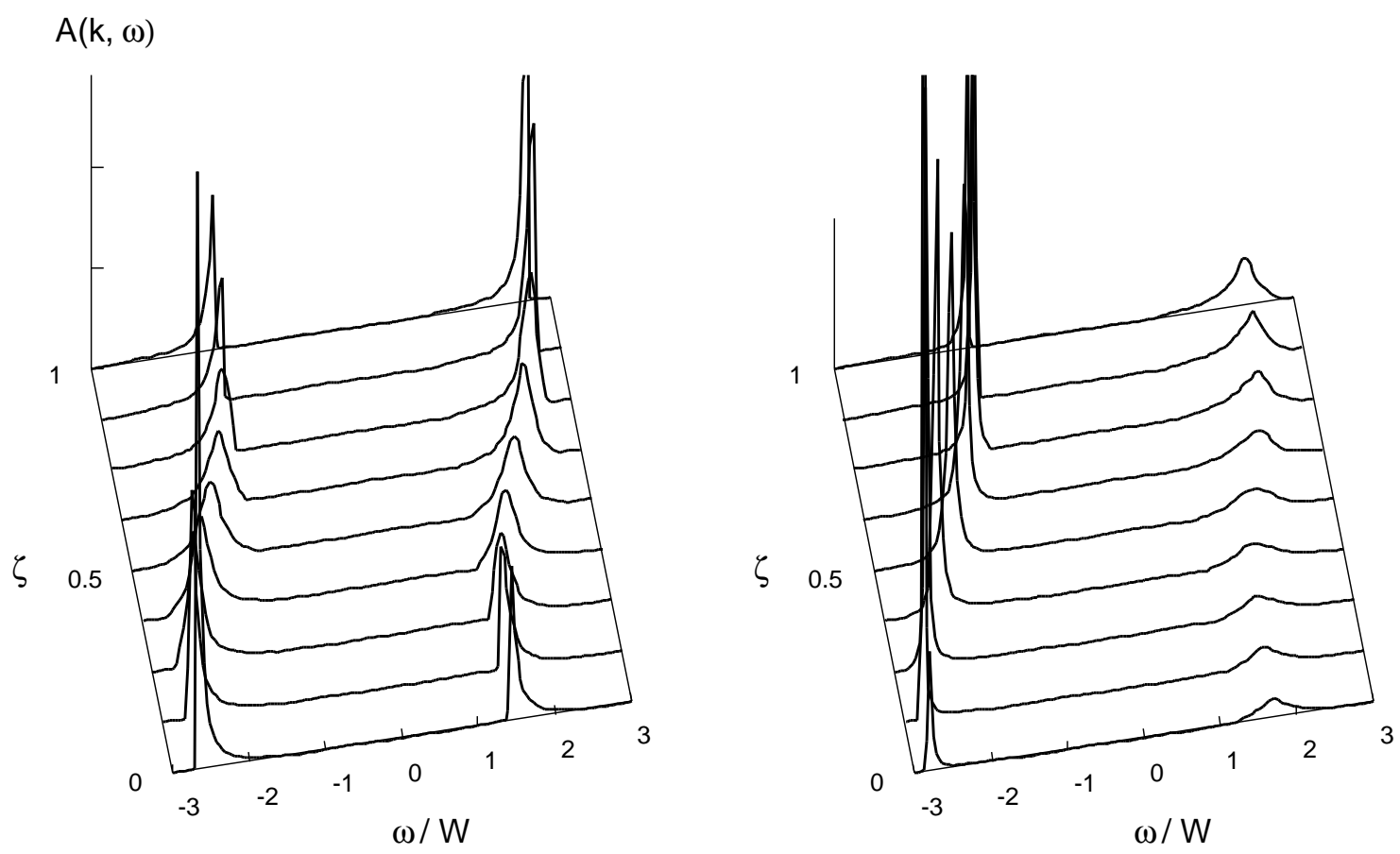

Figure 4: Spectral function $A_{\uparrow}(k, \omega)$ on a cubic lattice at $J_{\mathrm{H}} / W=2$ and $\mathrm{x}=0.3$, (left) in the paramagnetic phase $T=1.05 T_{\mathrm{c}}$, and (right) in the ferromagnetic phase $T=0.5 T_{\mathrm{c}}$. Here, $k / \pi=(\zeta, \zeta, \zeta)$.

where $Q=(\pi, \pi, \pi)$.

In Fig. 1 we show the quasiparticle excitation structure and its temperature dependence. There exists two-peak structure at around $\omega \sim \pm J_{\mathrm{H}}$. Above $T_{\mathrm{c}}$, peaks at upper and lower bands are symmetric and equally weighted. Below $T_{\mathrm{c}}$, the structure remains split but becomes asymmetric. For the up-spin electron, the integrated weight is transferred from upper band to lower band. We also see the change of the quasiparticle linewidth $\Gamma$. The lower band peak becomes sharper, which means the reduction of $\Gamma$ or the enlonged quasiparticle lifetime. On the other hand, $\Gamma$ for the upper band peak increases.

To investigate the change of the spectral weight in further detail, we calculate the DOS $A_{\sigma}(\omega)$ by $k$-integrating the spectral weight. In Fig. 5 we show the DOS as a function of temperature in the paramagnetic and ferromagnetic phases. Two subband structure at $\omega \sim \pm J_{\mathrm{H}}$ reflects the quasiparticle structure as described above. At the ground state, lower subband is composed of up-spin only, and down-spin band exists only at the high-energy region. The bandwidth of the DOS becomes narrower as temperature becomes higher. The band center is fixed at $\omega \sim \pm J_{\mathrm{H}}$ which is the energy level of the atomic limit $t=0$.

These results are in agreement with the exact result in a limiting case (Lorentzian DOS with $\left.J_{\mathrm{H}} \rightarrow \infty\right)$ discussed in $\$ 2.3$. Let us focus on the lower subband. The DOS is nearly proportional to the spin polarization of the localized spin, namely

$$
\begin{aligned}
& A_{\uparrow}(\omega) \sim z_{\uparrow}^{(l)}(M)=(1+M) / 2, \\
& A_{\downarrow}(\omega) \sim z_{\downarrow}^{(l)}(M)=(1-M) / 2,
\end{aligned}
$$

where $z$ is the quasiparticle weight discussed in $\S 2.3$. We see that $z$ is determined by the magnetization through $(1 \pm M) / 2$, which is the probability that the local spin is parallel to the itinerant electron with up (down) spin. This is easily understood from the nature of the double-exchange interaction which projects out the antiparallel component of the spins. This spin-dependent projection may be viewed as the evolution of the majority-minority band structure. The up (down) 

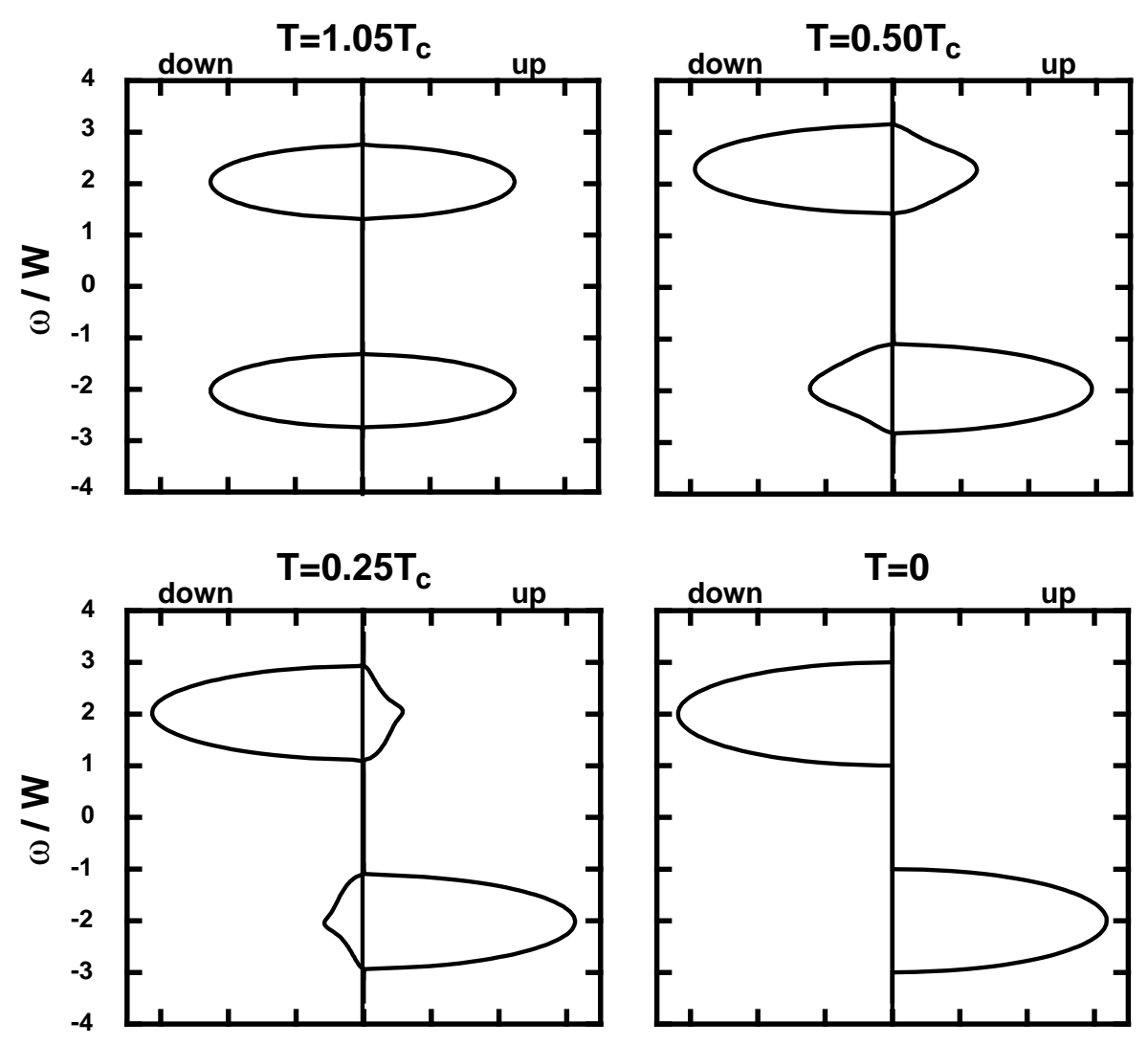

Figure 5: Temperature dependence of DOS for $J_{\mathrm{H}} / W=2$ and $x=0.3$, where $T_{\mathrm{c}}=0.019 \mathrm{~W}$. Peak structures are observed at $\omega \sim \pm J_{\mathrm{H}}$.

spin band becomes a majority (minority) band below $T_{\mathrm{c}}$ or under magnetic field. Shift of the spectral weight from the minority band to the majority band occurs as temperature is lowered. At the ground state $(M=1)$, minority band completely loses its weight.

Change of the electron bandwidth is understood qualitatively through Anderson-Hasegawa's picture. Electron hopping amplitude is proportional to $\cos (\theta / 2)$ where $\theta$ is the relative angle of the localized spins. At high temperature, $\theta$ deviates from zero due to spin fluctuation, and the amplitude of electron hopping matrix element and hence the bandwidth decreases. This is also shown by the virtual crystal approximation. 37

In order to account for the width of $A(k, \omega)$, or the quasiparticle lifetime, we have to go beyond a mean-field picture like in Anderson-Hasegawa approach. The origin of the linewidth $\Gamma$ is the thermal fluctuation of the spins. In the strong coupling region $J_{\mathrm{H}} \gg W$, spin scattering phenomena in the paramagnetic phase is so large that quasi-particles lose their coherence due to the inelastic scattering by thermally fluctuating spins. In the ferromagnetic phase, the spin fluctuation decreases as temperature is decreased. For the majority band, this decreases $\Gamma$, and in the limit $T \rightarrow 0$ the majority band becomes a free electron band. However, for the minority band, the spin projection causes further loss of the coherence, which leads to the increase of $\Gamma$ as well as the decrease of the quasiparticle weight $z$. Asymptotically in the limit $T \rightarrow 0, \Gamma$ approaches to a constant $\sim W$, and at the same time $z \rightarrow 0$. Then, at $T=0$, the minority band with finite $\Gamma$ is projected out.

Half metal. Metal with a DOS structure shown in Fig. 5 where only one of the spin species have the Fermi surface is called a half metal.38, (39) Namely, because of the 'Zeeman splitting' due to Strong Hund's coupling $J_{\mathrm{H}} \gg W$, ferromagnetic ground state of the DE model shows a perfect spin polarization and thus is a half-metal. Experimentally, spin-resolved photoemission investigation 
40.41) shows that the conduction band of the doped manganites is a half-metal. Artificial trilayer junction of manganites $\$ 2$ ) also shows a large tunneling magnetoresistance phenomena, and the spin polarization is estimated to be more than $80 \%$. Such a DOS structure creates a phenomena called tunneling magnetoresistance (TMR), which will be discussed in $\$ 5.1$.

Shift of the chemical potential. A direct consequence of the change of the bandwidth controlled by the magnetization will be observed in the shift of the chemical potential. In this case, the change of the DOS structure is in a way such that the band center is pinned by the Hund's coupling energy $\pm J_{\mathrm{H}}$ and the band edge shifts away from the center as magnetization is increased. Then, for a hole doped case the position of the chemical potential increases by increasing the magnetization.

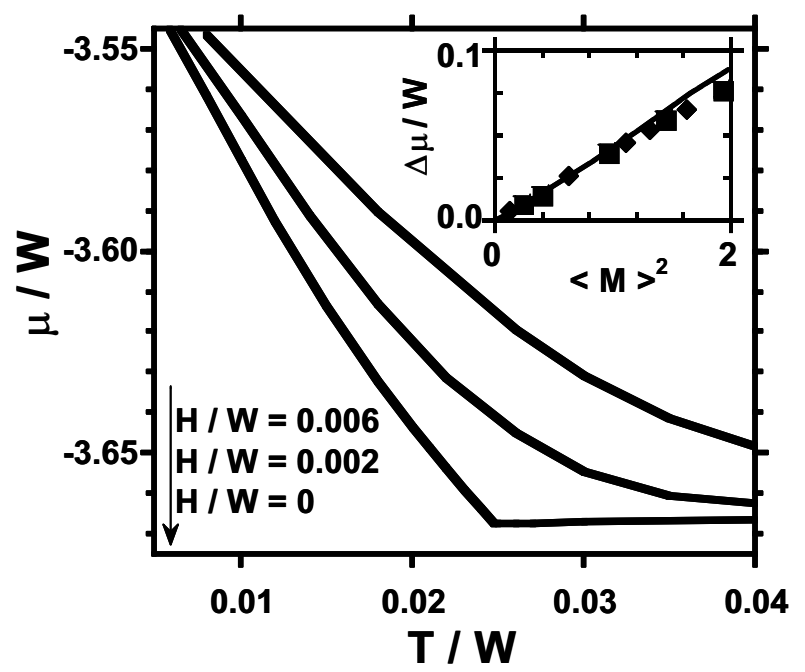

Figure 6: Temperature dependence of $\mu$ at $J_{\mathrm{H}} / W=4$ and $x=0.20$ under various magnetic field. Inset: $\Delta \mu / W$ as a function of $M^{2}$. Lines show the result at $T<T_{\mathrm{c}}$ for $H=0$. Squares and diamonds are data at $T=1.01 T_{\mathrm{c}}$ and $1.2 T_{\mathrm{c}}$ by applying $H$, respectively.

In Fig. 6 we show the temperature dependence of the chemical potential at $x=0.2$ under various magnetic field.13 At $H=0$, chemical potential $\mu$ is nearly temperature independent above $T_{\mathrm{c}}$. Below $T_{\mathrm{c}}, \mu$ shifts as a function of temperature. We also calculate $\mu$ and $M$, (i) at $H=0$ by changing temperature in the region $T \leq T_{\mathrm{c}}$, and (ii) at fixed temperature above $T_{\mathrm{c}}$ by changing $H$. In the inset of Fig. 6 we plot $\mu$ as a function of magnetic moment $M^{2}$ for both cases. As a result, we see the scaling relation

$$
\Delta \mu / W \propto M^{2}
$$

where $\Delta \mu \equiv \mu(T, H)-\mu\left(T=T_{\mathrm{c}}, H=0\right)$. We see that $\Delta \mu$ can be as large as $0.1 W$.

Thus, for a fixed band filling, the total change of the DOS width in the entire energy range causes the shift of $\mu$. The change in such a large energy scale controlled by magnetization produce the characteristic feature of the shift of $\mu$ in DE systems; namely, that the shift of $\mu$ is as large as a few tenth of $W$ and that the scaling relation (59) is satisfied up to such a large energy scale.

Such a large shift of $\mu$ might possibly be applied to electronic devices which controls the MOS gate voltage by the magnetic field.

\subsection{Magnetic structure and transport properties}

Magnetic transition temperature. In the limit $J_{\mathrm{H}} \rightarrow \infty$, Curie temperature $T_{\mathrm{c}}$ of the DE model is determined by the electron kinetic energy. Indeed, DMF calculation shows that $T_{\mathrm{c}}$ 
is scaled by electron hopping, i.e. $T_{\mathrm{c}} \propto W$ for $J_{\mathrm{H}}=\infty$ limit (see $\S 2.3$ ). Here we show the case for finite $J_{\mathrm{H}} / W$.

In Fig. 7 we show the Curie temperature $T_{\mathrm{c}}$ as a function of doping $x$ for various values of $J_{\mathrm{H}} / W$. At finite $J_{\mathrm{H}} / W, T_{\mathrm{c}}$ is reduced from $J_{\mathrm{H}}=\infty$ values. We also see that $T_{\mathrm{c}}$ systematically increase as $x$ is increased and have maximum at around $x \sim 0.5$, which is due to the increase of the kinetic energy.

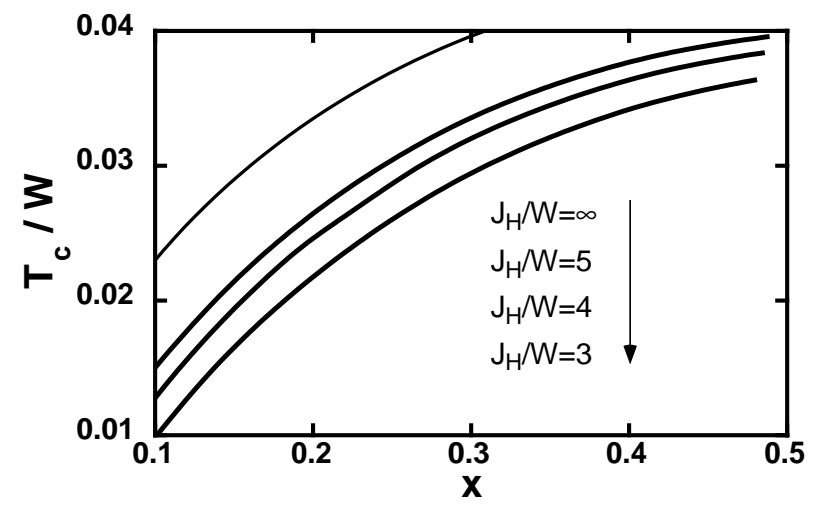

Figure 7: Curie temperature $T_{\mathrm{c}}$ as a function of $J_{\mathrm{H}} / W$ and $x$.

At half-filling $x=0$, there exists antiferromagnetic order. In Fig. 8 we show the phase diagram at $x=0$ for the $D=\infty$ Bethe lattice. In the weak coupling region $J_{\mathrm{H}} \ll W$, the Nèel temperature $T_{\mathrm{N}}$ is equivalent to the results from the SDW mean-field type equation with $J_{\mathrm{H}} / W$ dependence in an essential singular function. At $J_{\mathrm{H}} \gg W, T_{\mathrm{N}}$ is determined from the Heisenberg model with the exchange coupling $J_{\mathrm{AF}} \sim t^{2} / J_{\mathrm{H}} \propto W^{2} / J_{\mathrm{H}}$.

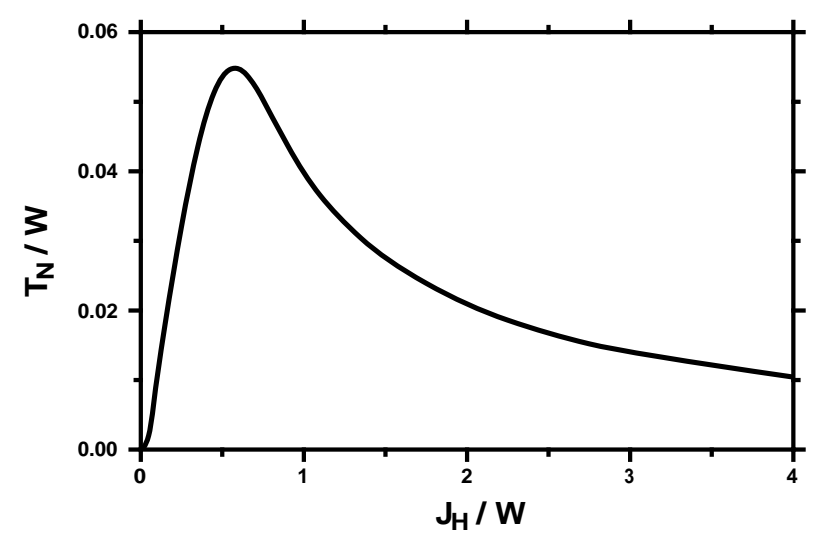

Figure 8: Nèel temperature at $x=0$.

Thus we see the antiferromagnetic order at $x=0$ and ferromagnetic ground state at sufficiently doped case. In the underdoped region $x \ll 1$, de Gennes discussed the existence of the canted state 3 . However, in $\$ 3.4$ we show that the canted state is unstable against phase separation, i.e. mixed phase of $x=0$ antiferromagnetic region and $x>0$ ferromagnetic region. 16 )

Resistivity as a function of magnetization. Resistivity $\rho(T)$ as well as total magnetization $M_{\text {tot }}$ as a function of temperature is given in Fig. 9. Here, $\rho_{0}$ is a constant of resistivity which corresponds to the Mott's limit value (inverse of the Mott's minimum conductivity) in three dimension. $M_{\text {sat }}$ is the saturated magnetization at $T=0$. 


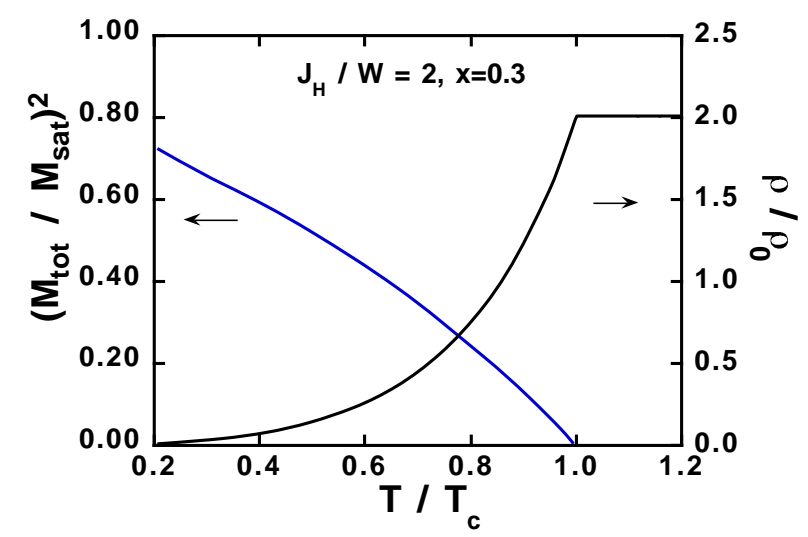

Figure 9: Resistivity $\rho$ and magnetization $M_{\text {tot }}$ as a function of temperature.

Above $T_{\mathrm{c}}$, the value of the resistivity is in the order of the Mott limit $\rho(T) \sim \rho_{0}$, with small $T$-dependence Below $T_{\mathrm{c}}$, resistivity drops quickly as magnetization increases. From the DMF calculation, , [1) it has been made clear that the resistivity behaves as

$$
\rho(M) / \rho(M=0)=1-C M^{2},
$$

where $C$ is a temperature/field independent constant. Namely, temperature and magnetic field dependences come from the magnetization $M=M(H, T)$. As a function of magnetization $M$, all the $T$ and $H$ dependent values $\rho(T, H)$ converge on a universal curve in (60). In other words, the origin of the resistivity is due to spin fluctuation, or more precisely spin disorder scattering, discussed by Kasuya 43 ) and later by Fisher and Langer. 44 )

In the Born approximation (weak coupling limit), we see $C=1.43$ ) A phenomenological treatment by Kubo and Ohata which estimates the resistivity from the spin fluctuation,

$$
\rho \propto(\delta S)^{2} \propto 1-M^{2}
$$

also gives $C=1$. However, in the DMF at $J_{\mathrm{H}} \gg W$, we have $C>1$ which indicates the strong coupling behavior. In the next section, the relation with experimental MR is discussed in more detail.

Above $T_{\mathrm{c}}$, small $T$-dependence in $\rho(T)$ is observed within the DMF treatment. This result might be an artifact of the approximation since the local spin fluctuation is saturated above $T_{\mathrm{c}}$.

\subsection{Charge and spin dynamics}

Optical conductivity. Temperature dependence of the optical conductivity is shown in Fig. 10. In the paramagnetic phase, the spectrum splits into two peaks due to the 2 -subband structure of the DOS. Namely, intraband particle-hole channel creates a Drude-like peak at $\omega \sim 0$, while interband channel creates a peak at around $\omega \sim 2 J_{\mathrm{H}}$. In the inset of Fig. 10, we show the weight of the interband process as a function of $1-M^{* 2}$. The integrated weight of the interband optical process at $\omega \sim 2 J_{\mathrm{H}}$ defined by

$$
S=\int_{\omega_{c}}^{\infty} \mathrm{d} \omega \sigma(\omega)
$$

where cutoff frequency is taken as $\omega_{\mathrm{c}}=J_{\mathrm{H}}$. We see a scaling relation

$$
S \propto 1-M^{* 2}
$$




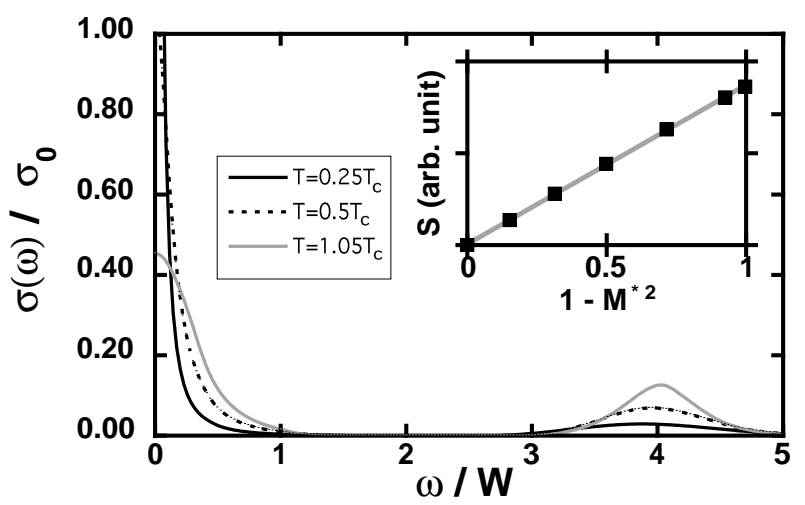

Figure 10: Temperature dependence of the optical conductivity for $J_{\mathrm{H}} / W=2$ and $x=0.3$. Inset: Integrated weight $S$ at high energy part $\omega \sim 2 J_{\mathrm{H}}$.

This is explained by the temperature dependence of DOS. Below Curie temperature, the DOS changes as in Fig. 5. Interband optical process at $\omega \sim 2 J_{\mathrm{H}}$ is constructed from a process of making a pair of lowerband hole and upperband electron. Then, the transfer of spectral weight by magnetization creates the change in the optical spectra as follows. For the up spin electrons, the spectral weight of lower and upper subbands are proportional to $\left(1+M^{*}\right) / 2$ and $\left(1-M^{*}\right) / 2$, respectively. Since this optical process conserves quasiparticle spin, the total weight of the optical spectrum is proportional to the product of the initial-state weight at lower subband and the finalstate weight at the upper subband, and hence scaled as $1-M^{* 2}$. Contribution from the down-spin band is also the same, and we have $S \propto 1-M^{* 2}$.

Stoner excitation. Stoner susceptibility is calculated by

$$
\chi(q, z)=\frac{1}{\beta N} \sum G\left(k+q, \mathrm{i} \omega_{n}+z\right) G\left(k, \mathrm{i} \omega_{n}\right) .
$$

Here, correlation effects are taken into account through the self-energy correction in $G$. In Fig. 11 we show $q$-dependence of $\operatorname{Im} \chi(q, \omega)$ for various temperatures, at $J_{\mathrm{H}} / W=2$ and $x=0.3$. We see two-peak structure at $\omega \sim 0$ and $\omega \sim 2 J_{\mathrm{H}}$ which is explained from the $J_{\mathrm{H}}$-split DOS structure. The Stoner absorption is produced from a particle-hole pair excitations with spin flip, which produces a peak at low energy from intra-band processes and another peak at $\omega \sim 2 J_{\mathrm{H}}$ from interband processes.

We see a weak $q$ dependence in the low frequecy part, especially at $T \ll T_{\mathrm{c}}$. This part of the Stoner process is dominated by the combination of the majority-minority quasiparticles. Since the quasiparticles in the minority band is incoherent, $\operatorname{Im} \chi(q, \omega)$ is weakly $q$ dependent. On the other hand, high energy part of $\operatorname{Im} \chi(q, \omega)$ at $T \ll T_{\mathrm{c}}$ have larger $q$ dependence. This part is dominated by the majority-majority quasiparticle channel. Thus $q$ dependence of $\operatorname{Im} \chi(q, \omega)$ reflects the band structure of the quasiparticles.

Let us see the $\omega$ dependence at the low frequency region. In Fig. 12 we show $\chi_{\mathrm{zB}}(\omega)=\chi(Q, \omega)$ where $Q=(\pi, \pi, \pi)$. We see that at small $\omega$ we have $\omega$-linear relation, i.e. $\operatorname{Im} \chi \propto \omega$ at $\omega \ll W$. Coefficients for $\omega$-linear part decrease by decreasing the temperature, and we find 4 )

$$
\operatorname{Im} \chi(Q, \omega) \propto\left(1-M^{* 2}\right) \omega
$$

for small values of $\omega$. The relation (65) is observed at all values of $q$ with weak $q$ dependence.

Low energy Stoner absorption is constructed from a minority particle and majority hole channel. Since the majority and the minority bands have the spectral weight proportional to $\left(1+M^{*}\right) / 2$ and $\left(1-M^{*}\right) / 2$, respectively, the low energy part of the Stoner absorption is proportional to their 

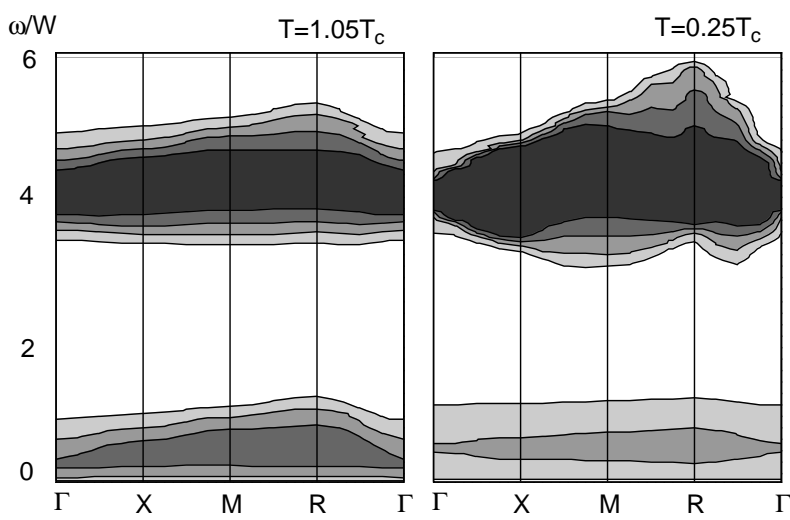

Figure 11: Contour plot of the Stoner absorption $\chi(q, \omega)$ on a cubic lattice at $J_{\mathrm{H}} / W=2$ and $x=0.3$, in the paramagnetic phase $T=1.05 T_{\mathrm{c}}$ (left) and in the ferromagnetic phase $T=0.25 T_{\mathrm{c}}$ (right). At $T=0$, the low energy part at $\omega \sim 0$ disappears.

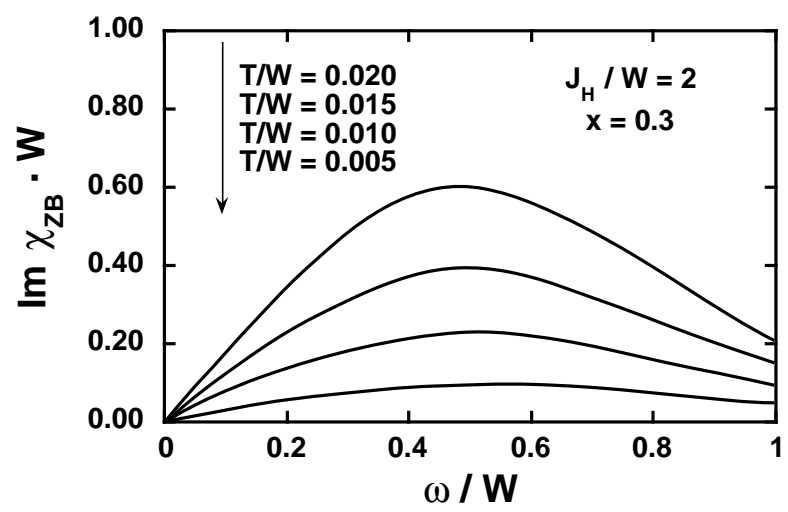

Figure 12: Stoner absorption $\chi(Q, \omega)$ at the zone boundary $Q=(\pi, \pi, \pi)$. At $J_{\mathrm{H}} / W=2$ and $x=0.3$, transition is at $T_{\mathrm{c}} / W=0.019$.

product, $1-M^{* 2}$. The $\omega$-linear behavior comes from the Fermi distribution function. The incoherence of the minority band gives the weak $q$ dependence. Thus we have the scaling relation $\mathcal{I}_{m} \chi \propto\left(1-M^{* 2}\right) \omega$. The weak $q$ dependence is in large contrast with the conventional weak ferromagnet where minority band is also coherent, which gives strong $q$ dependence through its band structure.

\subsection{Phase separation代}

Magnetic phase diagram of the weakly doped DE model has been studied by de Gennes. B) Assuming the homogeneity of the doped carriers, he concluded that the spin canted phase is the most energetically favorable state. However, we have recently shown that there exists an instability toward phase separation,16 and the assumption of uniformly doped charges by de Gennes is not valid.

One of the ways to discuss the phase separation is to make a grand canonical calculation of the particle number $x$ as a function of the chemical potential $\mu$. If there exists a jump of $x(\mu)$ at the critical value $\mu=\mu_{\mathrm{c}}$ in the thermodynamic limit, it implies that two phases with different doping $x$ coexist at $\mu=\mu_{\mathrm{c}}$. We have shown 16$)$ the jump of $x(\mu)$ in the DE model for sufficiently large

*Works shown here concerning the issue of phase separation has been made in collaboration with S. Yunoki, A. Moreo and E. Dagotto. 
$J_{\mathrm{H}} / W$. The jump occurs from $x=0$ to a state with finite $x$. The calculation has been performed within the DMF approach $(D=\infty)$, and cross checked by the Monte Carlo method for the $D=1$ and 2 clusters in the extrapolated limit of $T \rightarrow 0$.

Phase boundary is simply determined from the jump of $x(\mu)$. In Fig. 13 we show the $x-T$ phase diagram. At the low temperature region, we see mixed phases of $x=0 \mathrm{AF}$ state and doped $(x>0)$ state with either paramagnetic or ferromagnetic state, depending on temperature.

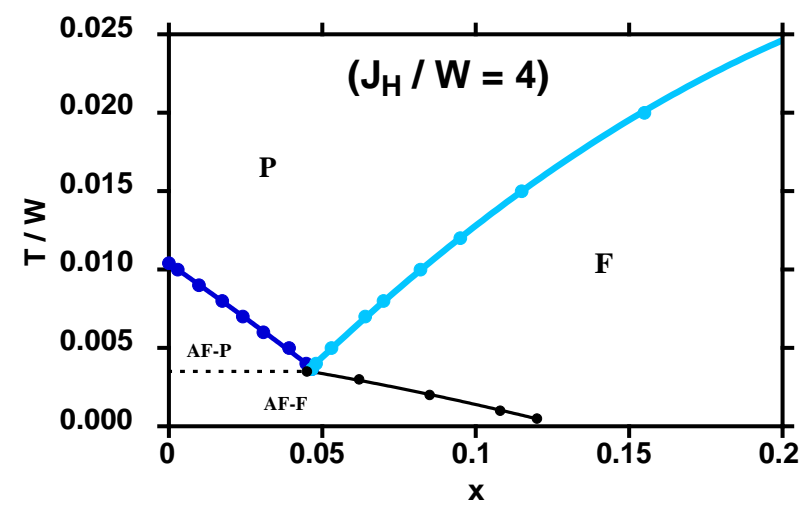

Figure 13: Phase diagram at $J_{\mathrm{H}} / W=4 . \mathrm{P}(\mathrm{F})$ represents paramagnetic (ferromagnetic) region. At $x=0$ we have antiferromagnetic (AF) phase. Regions labeled by AF-P (AF-F) are phase separated region with mixed phases of $\mathrm{AF}$ and $\mathrm{P}(\mathrm{AF}$ and $\mathrm{F}$ ) phases.

The mechanism of the phase separation may be understood as follows. In Fig. 14 we show the change of the density of states for different doping concentration. We see that the bandwidth substantially differs for different magnetic states, while the band center remains at $\omega \sim \pm J_{\mathrm{H}}$. As discussed in $\$ 3.1$, this typical DOS structure is a consequence of the DE half-metallic system. Let us consider the zero temperature limit. At the $x=0 \mathrm{AF}$ state, we have $\mu=0$. In order to hole dope this $\mathrm{AF}$ state, we need to decrease $\mu$ to a $\mathrm{AF}$ gap-value, $\mu=-\Delta_{\mathrm{AF}}$. However, as we see in Fig. 14, it is also possible to make a $\mathrm{F}$ state at $\mu=-\Delta_{\mathrm{AF}}$ with rich hole density $x$. AF state gains the exchange energy $\sim t^{2} / J_{\mathrm{H}}$, while the $\mathrm{F}$ state gains the kinetic energy $\sim t x$. Thus, in the limit $J_{\mathrm{H}} \gg t$, the doped $\mathrm{F}$ state becomes energetically favorable. In such a case, there exists the chemical potential $\mu_{\mathrm{c}}$ such that $-\Delta_{\mathrm{AF}}<\mu_{\mathrm{c}}<0$, where the energies of AF state with $x=0$ and $\mathrm{F}$ state with $x>0$ are equal. Level crossing from AF to $\mathrm{F}$ states occurs at this critical point $\mu=\mu_{\mathrm{c}}$. Doped $\mathrm{F}$ state is realized before doping the AF state. Thus we have a jump in the hole density from $x=0$ to finite $x$.

The discussion based on the DOS structure is quite generic. In strongly correlated systems with bistable phases, macroscopic change of the order parameters creates a large change in the electronic DOS. This gives a macroscopic jump in $\langle n\rangle$ from one phase to another when $\mu$ is fixed. Then, phase separation is associated with the density-driven phase transition.

Let us discuss the issue from a different viewpoint. In Fig. 15 we show the equal time spin correlation

$$
S(q)=\frac{1}{N} \sum_{i j}\left\langle\vec{S}_{i} \cdot \vec{S}_{j}\right\rangle \mathrm{e}^{\mathrm{i} q(i-j)}
$$

on a one dimensional system at $L=40, J_{\mathrm{H}} / W=4$ and $\beta=150 W^{-1}$. We clearly see the crossover from the antiferromagnetic state at $n=1$ with a peak of $S(q)$ at $q=\pi$, and the ferromagnetic state at $n \sim 0.7$ with the peak at $q=0$. Around $n \sim 0.9$, where large change of $n(\mu)$ is observed, we see two peak structure at $q=\pi$ and $q=0$. This result is consistent with mixed phase of ferromagnetic and antiferromagnetic states. On the other hand, magnetic states with incommensurate momentum, typically at $q=2 k_{\mathrm{F}}$, have been observed for the weak coupling region $J_{\mathrm{H}} \lesssim W$. Thus the tendency toward phase separation is prominent at the strong coupling region. 


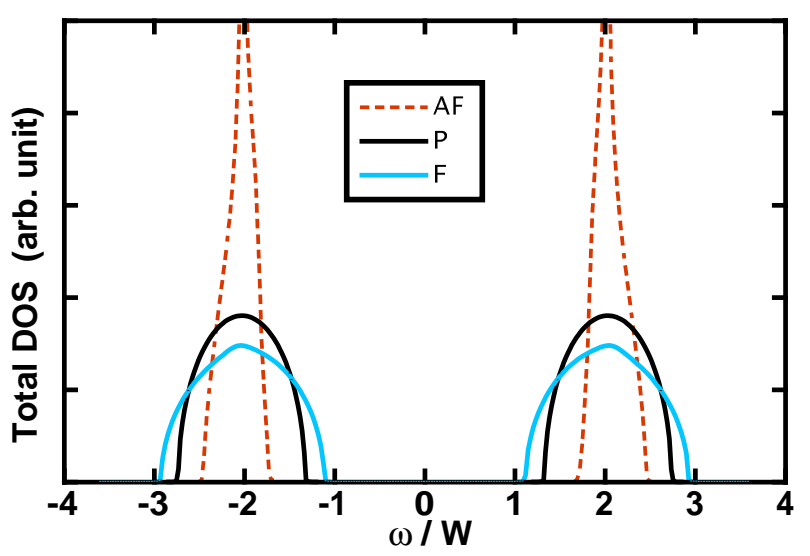

Figure 14: Density of states for ferromagnetic $(\mathrm{F})$, paramagnetic $(\mathrm{P})$ and antiferromagnetic (AF) states at $x=0.2,0.1$ and 0 , respectively, for $J_{\mathrm{H}} / W=2$ and $T / W=0.005$.

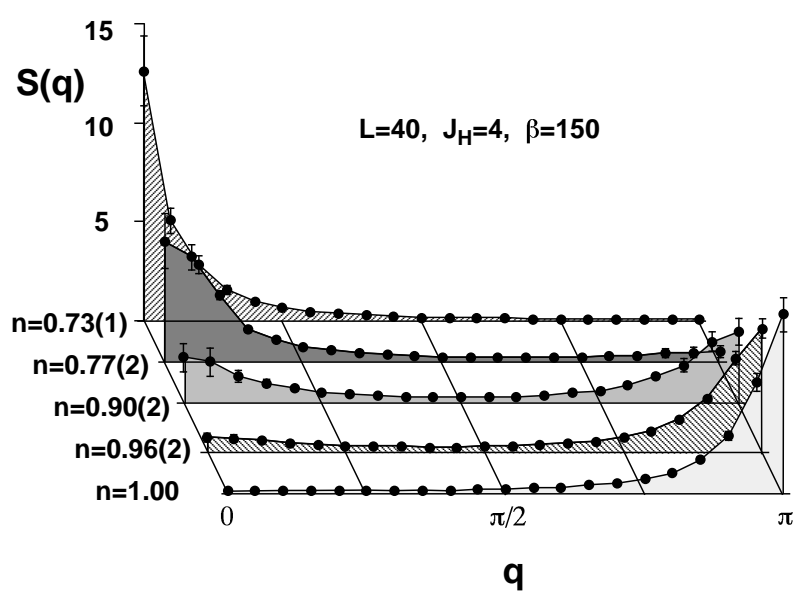

Figure 15: Equal time spin correlation for various electron concentration. Shaded area are guides to eyes. Error bars of the concentrations in the last digits are given in parenthesis.

Recently, the issue of phase separation is also investigated by various methods. $45,46,47)$ The model in the weak coupling region $J_{\mathrm{H}} \ll W$ has also been studied. 4 ) However, the mechanism of the phase separation might be different from the strong coupling region where the half-metallic DOS plays an important role. In manganites, several experiments claim the existence of phase separation.49.50) We will later discuss this issue in comparison with experiments in detail.

\section{1/S CORRECTIONS}

Spin wave expansion. We introduce a linear spin wave theory. The spin wave operators are introduced from

$$
S_{i}^{+} \simeq \sqrt{2 S} a_{i}, \quad S_{i}^{-} \simeq \sqrt{2 S} a_{i}^{\dagger}, \quad S_{i}^{z}=S-a_{i}^{\dagger} a_{i}
$$

Hereafter we restrict ourselves to the lowest order terms of the $1 / S$ expansion at $T=0$, where the localized spins are perfectly polarized so that $\left\langle a_{i}^{\dagger} a_{i}\right\rangle=0$. We consider the half-metallic ground state, i.e. $f_{k \downarrow}=0$ where $f_{k \sigma}$ is the Fermi distribution function. 

as 12 )

The spin wave self-energy in the lowest order of $1 / S$ expansion is obtained diagramatically

$$
\begin{aligned}
\Pi(q, \omega)= & -\frac{2 J_{\mathrm{H}}^{2}}{S} \frac{1}{N \beta} \sum_{k, n} G_{\uparrow}\left(k, \mathrm{i} \omega_{n}\right) G_{\downarrow}\left(k+q, \mathrm{i} \omega_{n}+\mathrm{i} \nu\right) \\
& -\frac{J_{\mathrm{H}}}{S} \frac{1}{N \beta} \sum_{k, n}\left(G_{\uparrow}\left(k, \mathrm{i} \omega_{n}\right)-G_{\downarrow}\left(k, \mathrm{i} \omega_{n}\right)\right) \mathrm{e}^{\mathrm{i} \omega_{n} 0_{+}} \\
= & \frac{1}{S N} \sum_{k} f_{k \uparrow}\left(J_{\mathrm{H}}-\frac{2 J_{\mathrm{H}}^{2}}{2 J_{\mathrm{H}}-\left(\omega+\varepsilon_{k}-\varepsilon_{k+q}\right)}\right) .
\end{aligned}
$$

Here, $G_{\sigma}\left(k, \mathrm{i} \omega_{n}\right)=\left(\mathrm{i} \omega_{n}-\varepsilon_{k}+\sigma J_{\mathrm{H}}\right)^{-1}$ is the fermion Green's function, and $\beta=1 / T$.

The spin wave dispersion relation $\omega_{q}$ is obtained self-consistently from $\omega_{q}=\Pi\left(q, \omega_{q}\right)$. We have

$$
\omega_{q}=\frac{1}{S N} \sum_{k} f_{k \uparrow}\left(J_{\mathrm{H}}-\frac{2 J_{\mathrm{H}}^{2}}{2 J_{\mathrm{H}}-\left(\varepsilon_{k}-\varepsilon_{k+q}\right)}\right)+O\left(1 / S^{2}\right),
$$

where $f_{k \uparrow}$ is the fermi distribution function of the majority band.

Let us consider the strong Hund coupling limit $J_{\mathrm{H}} \gg t$. If we assume a simple cubic lattice with nearest-neighbor electron hopping,

$$
\varepsilon_{k}=-2 t\left(\cos k_{x}+\cos k_{y}+\cos k_{z}\right)
$$

we have

$$
\omega_{q} \simeq E_{\mathrm{sw}} \frac{3-\cos q_{x}-\cos q_{y}-\cos q_{z}}{6}
$$

where $E_{\mathrm{sw}} \equiv \omega_{q=Q}-\omega_{q=0}$ is the spin wave bandwidth, given by

$$
E_{\mathrm{sw}}=\frac{6 t}{S N} \sum_{k} f_{k \uparrow} \cos k_{x}
$$

We see that in the strong coupling region the spin wave bandwidth is determined only by the electron transfer energy.

In the isotropic case, the spin stiffness is defined via $\omega_{q}=D q^{2}$ in the long wavelength limit $q \rightarrow 0$. From eq. (69) we have

$$
D=\frac{1}{2 S} \frac{1}{N} \sum_{k} f_{k \uparrow}\left[\frac{1}{2} \frac{\partial^{2} \varepsilon_{k}}{\partial k^{2}}-\frac{1}{2 J_{\mathrm{H}}}\left(\frac{\partial \varepsilon_{k}}{\partial k}\right)^{2}\right] .
$$

We emphasize that the expansion given here is not with respect to $J_{\mathrm{H}} /(t S)$ but to $1 / S$, due to energy denominator $2 J_{\mathrm{H}}$ between up- and down- spin electrons. This is understood from the fact that $J_{\mathrm{H}}$ plays an role of a projection and does not enter the energy scale by itself in the limit $J_{\mathrm{H}} \rightarrow \infty$. Therefore, the calculation is valid even in the large Hund's coupling limit $J_{\mathrm{H}} \gg t$ as long as we restrict ourselves to $T=0$. Indeed, the result for $J_{\mathrm{H}} \rightarrow \infty$ obtained by the present approach is equal to those in the projection limit $J_{\mathrm{H}}=\infty$ shown by Kubo and Ohata.17)

Discussion. In the DE model with $J_{\mathrm{H}} \gg W$ we observe a short-range spin interaction, in contradiction with the case of $J_{\mathrm{H}} \ll W(s-d$ model $)$ where the well-known RKKY interaction is long ranged with a power-law decay. The difference comes from the electronic structure.

In the case $J_{\mathrm{H}} \ll W$ where electronic DOS is the ordinary one (not half-metal), the gapless quasi-particle excitation of the electronic part creates a particle-hole spin excitation channel with 
$2 k_{\mathrm{F}}$ singularities. Interactions among localized spins are mediated by this gapless channel and thus long ranged.

On the other hand, for the DE model with $J_{\mathrm{H}} \gg W$ the half-metallic DOS creates a gap due to $J_{\mathrm{H}}$ splitting, and unlike the former case the particle-hole spin channel is massive and short ranged. The qualitative difference comes from the half-metallic structure. In the real-space picture, we consider a perfectly spin polarized state at $T=0$ and twist a spin at site $i_{0}$. Spin polarization of itinerant electrons are along the total polarization axis except for the $i_{0}$-th site where it orients toward the local spin direction $\vec{S}_{i_{0}}$. In the strong coupling limit $J_{\mathrm{H}} \gg W$, the electron at site $i_{0}$ is localized because it has different spin orientation from the spins in neighboring sites. Since the effective interaction between localized spins are mediated by the motion of electrons, the effective spin-spin interaction is short ranged. As $J_{\mathrm{H}} / W$ increases, electrons become more localized so the range of effective interaction becomes shorter. In the extreme limit $J_{\mathrm{H}} \rightarrow \infty$, the interaction is nonzero only for the nearest neighbors which gives a cosine-band dispersion.

Let us discuss the higher orders of $1 / S$ expansion terms. In an ordinary (insulating) Heisenberg ferromagnet, the first $1 / S$ term is the relevant term with respect to the one-magnon dispersion $\omega_{q}$. Higher orders of $1 / S$ expansion only give magnon-magnon interaction terms, and thus irrelevant within the one magnon Hilbert space. However, for the DE model, higher order terms also give one-magnon kinetic terms. We need to take into account the asymptotic $1 / S$ expansions even for the one-magnon dispersion relations. Such higher order terms may be considered as vertex corrections to the self-energy term $\Pi(q, \omega)$.

Numerically, Kaplan et al.51) studied the $S=1 / 2$ case at $J_{\mathrm{H}} \rightarrow \infty$. They observed cosine-band type behaviors in the well-doped cases, and the deviation from them in the limit $n \rightarrow 0$ and $n \rightarrow 1$. The result might be understood from the Migdal's discussion. Let us consider the electron kinetic energy $E_{\text {kin }}$ and the energy scale of magnons $\left\langle\omega_{q}\right\rangle$ of the DE model. The deviation from a cosineband comes from the vertex correction which is relevant if $E_{\text {kin }} \lesssim\left\langle\omega_{q}\right\rangle$, which occurs in the lightly hole/electron doped region $n \rightarrow 1$ or $n \rightarrow 0$. On the other hand, in the well-doped region we have $E_{\text {kin }} \gtrsim\left\langle\omega_{q}\right\rangle$ and the vertex corrections are small. In the region where doped manganites show ferromagnetism, vertex corrections do not seem to be important. This explains the consistency between experiments and $1 / S$ results

\section{COMPARISON WITH EXPERIMENTS}

Abbreviations: Hereafter we use these abbreviations: $\mathrm{La}_{1-x} \mathrm{Sr}_{x} \mathrm{MnO}_{3}$ (LSMO), $\mathrm{La}_{1-x} \mathrm{~Pb}_{x} \mathrm{MnO}_{3}$ (LPMO), $\mathrm{La}_{1-x} \mathrm{Ca}_{x} \mathrm{MnO}_{3}$ (LCMO), $\mathrm{Pr}_{1-x} \mathrm{Sr}_{x} \mathrm{MnO}_{3}$ (PSMO), $\mathrm{Nd}_{1-x} \mathrm{Sr}_{x} \mathrm{MnO}_{3}$ (NSMO), $\mathrm{Pr}_{1-x} \mathrm{Ca}_{x} \mathrm{MnO}_{3}$ (PCMO).

\subsection{Experimental -varieties of properties in "CMR manganites"-}

Let us briefly mention the varieties of phenomena in CMR manganites. For a review of recent experiments, readers are referred to ref. 77. It is emphasized that systematic studies of A-site substitution is quite important to understand the complex behaviors in manganites. Extrinsic effects due to grain/domain boundaries are also discussed.

A-site substitution. Recent improvements in precise control of the A-site cations substitutions in $\mathrm{AMnO}_{3}$ revealed a complex phase diagram as a function of substitution, temperature and magnetic field. They exhibit various phases with magnetic, charge, orbital and lattice orderings. For example, phase diagram for doping $(x)$ vs. temperature $(\mathrm{T})$ is well known for $\mathrm{La}_{1-x} \mathrm{Ca}_{x} \mathrm{MnO}_{3}$ (LCMO), 52) as well as $\mathrm{La}_{1-x} \mathrm{Sr}_{x} \mathrm{MnO}_{3}$ (LSMO), ${ }_{53} \mathrm{Nd}_{1-x} \mathrm{Sr}_{x} \mathrm{MnO}_{3}$ (NSMO) and $\operatorname{Pr}_{1-x} \mathrm{Ca}_{x} \mathrm{MnO}_{3}$

(PCMO).54) Effects of A-site substitution is also studied for a fixed doping.55,56,57)

Major effects of A-site substitution are the bandwidth control and the carrier number control. It is well understood that the ratio of rare-earth $(3+)$ ions and alkaline-earth $(2+)$ ions determines 


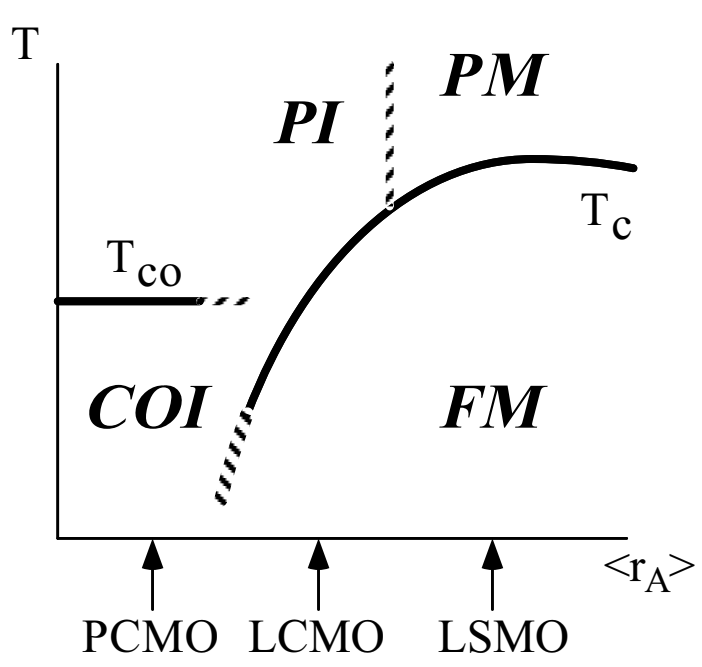

(a)

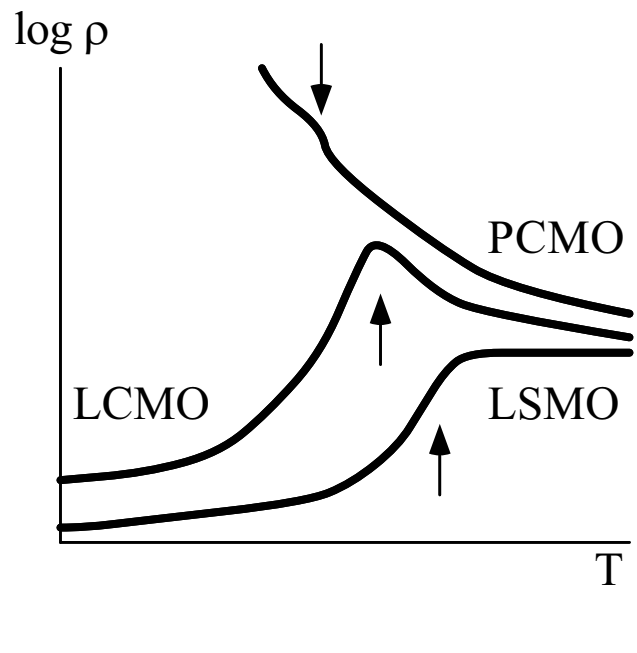

(b)

Figure 16: (a) Schematic phase diagram at $x \sim 1 / 3$ by ionic radius of A-site $\left\langle r_{\mathrm{A}}\right\rangle$. Abbreviations are: paramagnetic metal $(\mathrm{PM})$, paramagnetic insulator $(\mathrm{PI})$, ferromagnetic metal $(\mathrm{FM})$, charge ordered insulator $(\mathrm{COI})$, as well as Curie temperature $\left(T_{\mathrm{c}}\right)$ and charge ordering temperature $\left(T_{\mathrm{CO}}\right)$. (b) Qualitative behaviors in $\rho(T)$ for (La,Sr) $\mathrm{MnO}_{3}$ (LSMO), $(\mathrm{La}, \mathrm{Ca}) \mathrm{MnO}_{3}(\mathrm{LCMO})$ and $(\mathrm{Pr}, \mathrm{Ca}) \mathrm{MnO}_{3}(\mathrm{PCMO})$ at $x \sim 1 / 3$. Uparrows $(\uparrow)$ in the figure show $T_{\mathrm{c}}$, while downarrows $(\downarrow)$ indicates $T_{\mathrm{CO}}$.

the nominal values of the carrier number $x$. At the same time, change of the average radius of the A-site ions $\left\langle r_{\mathrm{A}}\right\rangle$ by chemical substitutions gives the "bandwidth control" through the chemical pressure. Such kind of chemical control creates a large change in the nature of the compounds. In general, compounds with larger $\left\langle r_{\mathrm{A}}\right\rangle$ have higher $T_{\mathrm{c}}$.55,56,57 It is considered to be due to wider effective bandwidth for $e_{\mathrm{g}}$ electrons in larger $\left\langle r_{\mathrm{A}}\right\rangle$ compounds, since it gives less Mn-O octahedra tilting.

However, we should note that it is still controversial whether the phase diagram is controlled mostly by the bandwidth alone. For example, ionic size variation $\sigma\left(r_{\mathrm{A}}\right)$ also plays some role to change $T_{\mathrm{c}}, 58$ as well as the fact that decrease of $T_{\mathrm{c}}$ for small $\left\langle r_{\mathrm{A}}\right\rangle$ is substantially larger than the estimated value from the change of bond angles.

Let us concentrate on the region $x \sim 1 / 3$ where it is far from antiferromagnetic insulating phase at $x \sim 0$ and the region with charge and orbital ordering at $x \sim 0.5$. The compounds are roughly classified as follows:

- High $T_{\mathrm{c}}$ compounds: e.g. LSMO.

A canonical example for the high $T_{\mathrm{c}}$ compounds is (La,Sr) $\mathrm{MnO}_{3}$ (LSMO) with $T_{\mathrm{c}} \sim 380 \mathrm{~K}$. Resistivity shows a small value at lowest temperature $\left(\rho_{0} \sim 10^{2} \mu \Omega \mathrm{cm}\right)$. At $T \sim T_{\mathrm{c}}, \rho(T)$ takes much larger value but still in the order of Mott's limit $\rho=2 \sim 4 \mathrm{~m} \Omega \mathrm{cm}$. Above $T_{\mathrm{c}}$, $\rho(T)$ shows a metallic behavior, i.e. $\mathrm{d} \rho(T) / \mathrm{d} T>0$. Namely, this compound is a good metal below $T_{\mathrm{c}}$ and become an incoherent metal above $T_{\mathrm{c}}$ with the absolute value for $\rho(T)$ being near Mott's limit.53,59)

- Low $T_{\mathrm{c}}$ compounds: e.g. LCMO, PSMO.

LCMO is the most well-investigated compound. Ca substitution creates smaller $\left\langle r_{\mathrm{A}}\right\rangle$ and larger $\sigma\left(r_{\mathrm{A}}\right)$. It has lower $T_{\mathrm{c}} \sim 280 \mathrm{~K}$ compared to LSMO, and shows metal to insulator transition at around $T_{\mathrm{c}}$.52, 6 , 60)

- Compounds with charge ordering instability: e.g. PCMO. 
As $\left\langle r_{\mathrm{A}}\right\rangle$ is further decreased, compounds show a charge ordering at $T \sim 200 \mathrm{~K}, 61,04$, , 62, , 8 ) In the zero-field cooling process, ferromagnetic metal phase does not appear.

At $x \sim 1 / 2$, the phase diagram becomes more complex. At the lowest temperature, the tendency of the competition between ferromagnetism and charge ordering 63, 64 driven by $\left\langle r_{\mathrm{A}}\right\rangle$ control remains the same. It has been recently discovered that in the intermediate region, anew phase of A-type antiferromagnetic metal region exists in the narrow vicinity of $x=1 / 2.65$, 66) Temperature dependence is also complex. Behaviors of the resistivity above $T_{\mathrm{c}}$ are roughly the same with the case of $x \sim 1 / 3$. For example, metallic behavior above $T_{\mathrm{c}}$ is observed for NSMO at $x=1 / 2$ as well as compounds with larger $\left\langle r_{\mathrm{A}}\right\rangle$. Substitution of $\mathrm{Nd}$ by Sm reduces $\left\langle r_{\mathrm{A}}\right\rangle$ and makes the paramagnetic phase insulating.67

Extrinsic effects in polycrystal samples. In samples with multiple grain structures, it has been shown that there exist so-called tunneling magnetoresistance (TMR) phenomena through the spin valve mechanism. 60, 68, 69, (6) Magnetoresistance of the material with artificially controlled grain/interface boundaries are also studied to realize a low-field MR device through TMR. $42,41,72)$

In manganites, the half-metallic behavior due to $\mathrm{DE}$ interaction is considered to cause a large amplitude in MR. Spin polarizations in grains are schematically depicted in Fig. 17. In the zero field case, each grain have different spin orientations. Namely, the spins of the half-metallic electrons are different from one grain to another. Due to the nearly perfect polarization nature of itinerant electrons, inter-grain hopping amplitude is suppressed by such random spin polarizations. Under the magnetic field, spin polarization of grains become parallel to the external field. Intergrain electron hopping becomes larger in this case. For multi-grain system with half-metallic states such spin valve phenomena becomes prominent and gives MR effect in a low field range.

Resistivity in polycrystal samples seems to be dominated by such extrinsic effects. One should be careful about discussing the experimental data from a microscopic point of view. Such TMR behavior in these perovskite manganites should be discussed in relation with other half-metallic materials such as $\mathrm{CrO}_{2}$ or $\mathrm{Tl}_{2} \mathrm{Mn}_{2} \mathrm{O}_{7}, 39$, (73)

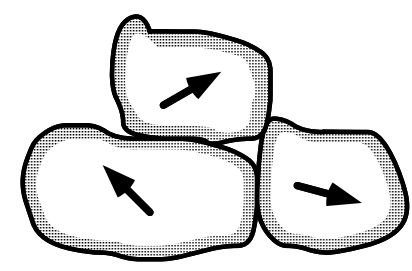

$\mathbf{H}=\mathbf{0}$

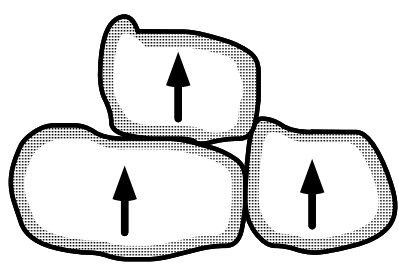

$\mathbf{H}>\mathbf{0}$

Figure 17: Grains of the polycrystal samples. (a) at zero magnetic field $H=0$, (b) under magnetic field $H>0$. Arrows indicate the orientation of the magnetization for each grains. For the relation with low field TMR, see the text.

\subsection{Comparison with high Curie temperature compounds}

Here, we will show the comparison of theoretical results with experimental data of high $T_{\mathrm{c}}$ compounds such as LSMO and LPMO. We discuss that the DE Hamiltonian alone explains most of the thermodynamics of these manganites, including Curie temperature and resistivity. 
Curie temperature. In Fig. 18 we plot the $x$ dependence of the Curie temperature for LSMO, together with the fitting curves obtained by DMF. From the fitting, we see that with parameters $W \sim 1 \mathrm{eV}$ and $J_{\mathrm{H}} / W \sim 4$ the value of $T_{\mathrm{c}}$ as well as its $x$-dependence is reproduced. The bandwidth of $W \sim 1 \mathrm{eV}$ is a typical value for 3 d transition metal oxides, and is consistent with the band calculation estimate for manganites.74, 75, 76) It is also consistent with the value obtained from the spin wave dispersion fit, 12 ) shown later in this section.

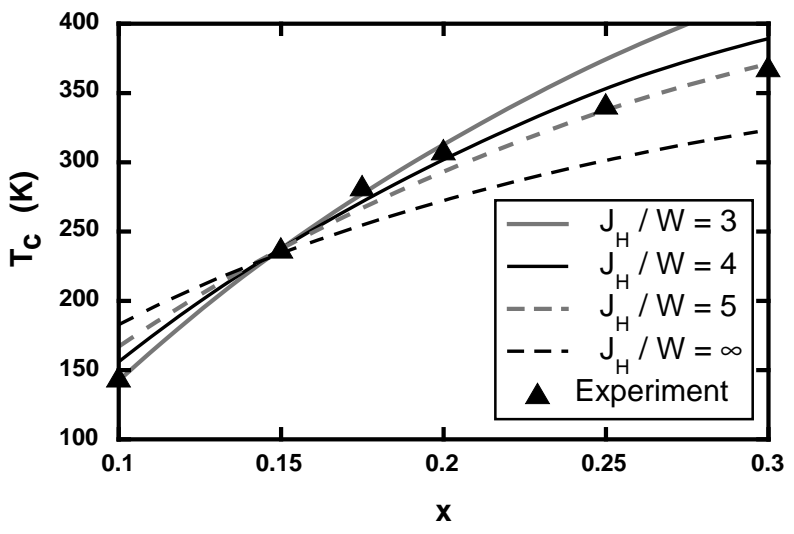

Figure 18: Curie temperature $T_{\mathrm{c}}$ of the DE model in comparison with those for $\mathrm{La}_{1-x} \mathrm{Sr}_{x} \mathrm{MnO}_{3}$. Experimental data are from ref. 77 .

Thus the value of $T_{\mathrm{c}}$ in LSMO is reproduced by the DE model alope. This result is consistent with other methods, e.g. high-temperature expansion by Röder et al. .78 as well as by the Monte Carlo method. (16)

Millis et al.6) discussed that the magnitude of $T_{\mathrm{c}}$ as well as its $x$ dependence for LSMO cannot be accounted for by the DE model alone, and discussed the importance of the dynamic Jahn-Teller effect. However, this part of their discussion is due to an inappropriate estimate of $T_{\mathrm{c}}$ which is based on a calculation of effective spin coupling at $T=0$. In the itinerant systems, $T_{\mathrm{c}}$ is reduced by spin fluctuation, in general. Although $T_{\mathrm{c}}$ scales with $W$, it is quite small compared to $W$ due to the prefacter, whose typical value is $T_{\mathrm{c}} \lesssim 0.05 \mathrm{~W}$ (See Fig. 2). Thus bandwidth of $1 \mathrm{eV}$ creates $T_{\mathrm{c}}$ of the order of room temperature. Decrease of $T_{\mathrm{c}}$ as $x$ decreases is explained by the reduction of the kinetic energy 34

Resistivity and magnetoresistance. Resistivity of the high- $T_{\mathrm{c}}$ compounds in single crystals at sufficiently large doping $x \sim 1 / 3$ are different from those of low- $T_{\mathrm{c}}$ compounds or polycrystal samples. For LSMO at $x \sim 1 / 3,53$ residual resistivity $\rho_{0}$ is in the order of a few $10 \mu \Omega \mathrm{cm}$, and the temperature dependence $\rho(T)$ shows a monotonously increase, i.e. $\mathrm{d} \rho / \mathrm{d} T>0$ even above $T_{\mathrm{c}}$. (For LPMO, see ref. 79. Recent experiment by Cheong et al.59) shows that the resistivity of LSMO continuously increase up to $1000 \mathrm{~K}$, without saturation or metal-semiconductor transition.) The value of resistivity at $T_{\mathrm{c}}$ is typically $\rho\left(T_{\mathrm{c}}\right)=2 \sim 4 \mathrm{~m} \Omega \mathrm{cm}$, which is in the order of the Mott limit. In short, LSMO is a good metal at $T \ll T_{\mathrm{c}}$, and an incoherent metal at $T \gtrsim T_{\mathrm{c}}$. The DE model reproduces these data (See Fig. 9). Similar temperature dependences of resistivity are observed in a wide class of materials of half-metals such as $\mathrm{CrO}_{2}$ and Heusler alloys.39)

It has been discussed that the DE model cannot explain the resistivity of LSMO in its absolute value as well as the temperature dependence.6) However, it is now clear that if one compares data for a high quality single crystal of LSMO (not polycrystal, or other compounds with lower $T_{\mathrm{c}}$ ), DE alone does account for the resistivity.

In Fig. 19 we show magnetoresistance of LSMO at $x=0.175$. Universal behavior of the magnetoresistance in the form

$$
-\Delta \rho / \rho_{0}=C M^{* 2}
$$




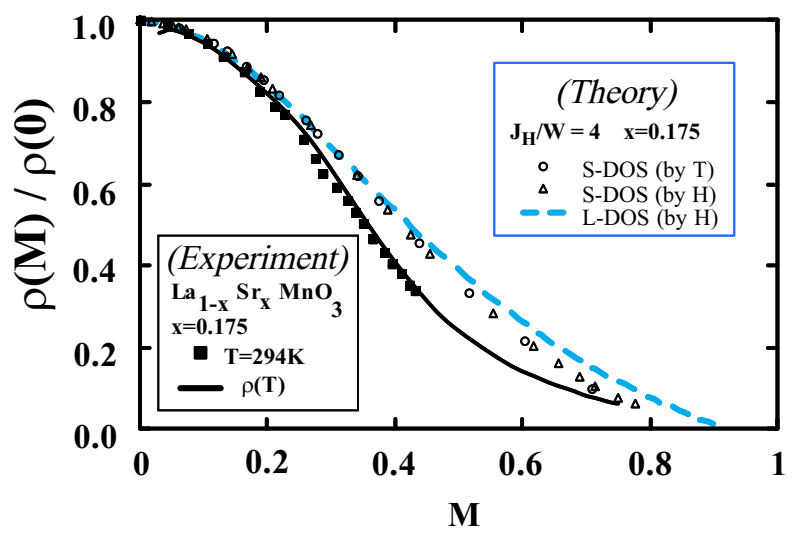

Figure 19: Magnetoresistance, plotted by magnetization vs. resistivity. Solid curves and filled symbols are the data for $\mathrm{La}_{0.875} \mathrm{Sr}_{0.175} \mathrm{MnO}_{3}$ from ref. 77. Open symbols and grey curve show DMF results for $J_{\mathrm{H}} / W=4$ and $x=0.175$, for semicurcular (S-DOS) and Lorentzian (L-DOS).

is observed, where $C \sim 4$ is temperature/field independent constant. 53, 77) Namely, the resistivity is directly related to the magnetization. In Fig. 19 we also show the DMF results. The curve reproduces the experimental data.11) The relation $\Delta \rho / \rho(0)=-C M^{2}$ is obtained theoretically in eq. (60). The value $C>1$ shows that the system is in the strong coupling region.

Note that in LCMO polycrystal samples, a different scaling relation

$$
\rho(M)=\rho(0) \exp \left(-\alpha M^{2} / T\right)
$$

has been reported 8 ) From such an activation-type temperature dependence, polaronic origin of the resistivity has also been discussed. The difference between the scaling relation in LSMO (74) and LCMO (75) indicates the qualitative difference for the origin of CMR.

Thermoelectric power. Seebeck coefficient $S$ for LSMO has been reported $\$$ They show non-universal behaviors, including the change of the sign. However, in the vicinity of $T_{\mathrm{c}}$, a scaling behavior in the form $-\Delta S / S(0) \simeq-\Delta \rho / \rho(0)$ irrespective of doping is reported, where $\Delta S$ and $\Delta \rho$ are the change of Seebeck coefficient and resistivity under magnetic field, and $S(0)$ and $\rho(0)$ are their zero-field values, respectively. 11 In Fig. 20 we plot the DMF result for the Seebeck coefficient $S$ on a Lorentzian DOS at $J_{\mathrm{H}} / W=4$. The data is plotted in the form $-\Delta S / S(0)$ vs. $-\Delta \rho / \rho(0)$. We see $-\Delta S / S(0) \simeq-\Delta \rho / \rho(0)$ for different values of $x$.

Spin excitation. From the neutron inelastic scattering experiment, spin wave dispersion relation of $\mathrm{La}_{0.7} \mathrm{~Pb}_{0.3} \mathrm{MnO}_{3}$ (LPMO at $\left.x=0.3\right)$ is investigated. 22 ) LSMO in the ferromagnetic metal region has also been studied $14,83,84,85$

Perring et al.22) found that the experimental data of spin wave dispersion relation for LPMO fits the cosine band form. They phenomenologically argued that a ferromagnetic Heisenberg model with nearest-neighbor spin exchange couplings $2 J_{\text {eff }} S \sim 8.8 \mathrm{meV}$ is a good candidate for the effective spin Hamiltonian, although the material is a metal.

Now we discuss the DE model results. 12, 51, 86 In Fig. 21, we plot the theoretical results together with the neutron inelastic scattering experiment data. As we have discussed previously, cosineband dispersion is obtained in the limit $J_{\mathrm{H}} \rightarrow \infty$. This gives the identical fit with the analysis by Perring et al., and the fitting parameter determines electron hopping as $t \sim 0.3 \mathrm{eV}$. Here, finite gap at $q=0$ is artificially introduced as done in ref. 82, due to some experimental inaccuracies.

At the zone boundary $q \sim(\pi, \pi, \pi)$ we see the deviation from the cosine band, or softening of the dispersion. The data is well accounted for by introducing some finite value for $J_{\mathrm{H}} / t$. In 


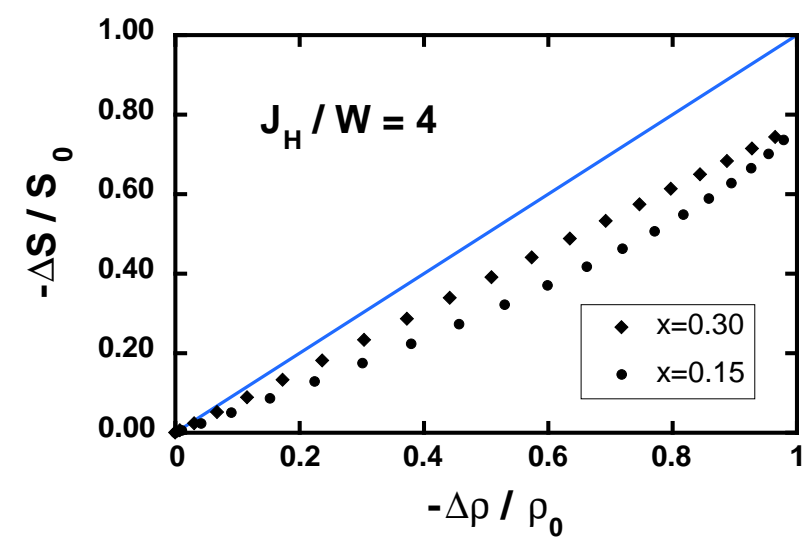

Figure 20: Seebeck coefficient under magnetic field, scaled by resistivity. The line is a guide to eyes.

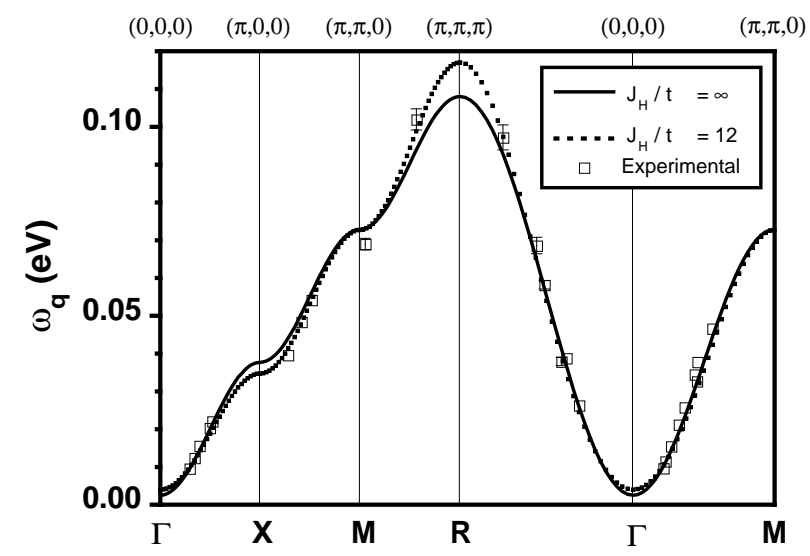

Figure 21: Spin wave dispersion at $x=0.3$. The experimental data for $\mathrm{La}_{0.7} \mathrm{~Pb}_{0.3} \mathrm{MnO}_{3}$ is from ref. 82 .

Fig. 21 we show the result for $J_{\mathrm{H}} / t=12$. From fitting we obtain $t \sim 0.26 \mathrm{eV}$ and $J_{\mathrm{H}} \sim 3.1 \mathrm{eV}$. Although it is not easy to estimate the systematic errors of the fitting, it is plausible to say that the DE model with $t=0.2 \sim 0.3 \mathrm{eV}$ and $J_{\mathrm{H}} \gtrsim 3 \mathrm{eV}$ explains the spin wave dispersion relation of LPMO. These values are the effective hopping energy of the double-exchange model with reduced degrees of freedom. Nevertheless, these values of $t$ are consistent with those estimated from the band calculation.7, 75,6 ,

Anomalous damping of zone boundary magnons are also reported.82) Within the DE model, spin wave excitation at finite temperature interacts with the Stoner continuum as shown in Fig. 12. From eq. (65), we see that

$$
\Gamma(q, T) \propto\left(1-M^{* 2}\right) \omega_{q},
$$

where $\Gamma(q, T)$ is the linewidth (inverse lifetime) of the magnon. This explains that the magnons are damped at finite temperature, especially at the zone boundary. Indeed such a scaling relation (76) is observed in LSMO.14)

Optical conductivity. Optical conductivity for $\mathrm{La}_{0.6} \mathrm{Sr}_{0.4} \mathrm{MnO}_{3}$ by Moritomo et al. .77) is shown in Fig. 22(a). Here, temperature independent part which is discussed to be due to $d-p$ charge-transfer type excitation is subtracted. There exists a peak at $\omega \sim 3 \mathrm{eV}$, and its temperature dependence is in a way that it vanishes at $T \rightarrow 0$. 

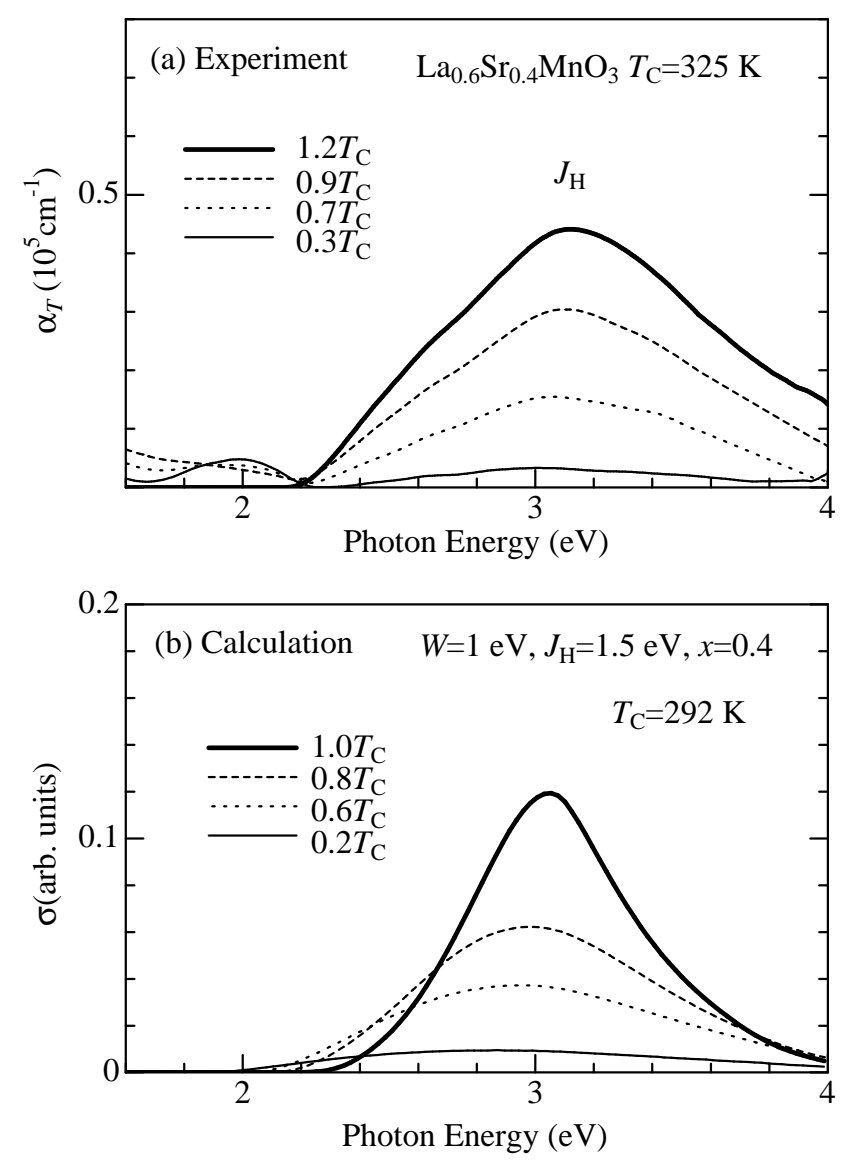

Figure 22: Optical conductivity. (a) Experimental data for $\mathrm{La}_{0.6} \mathrm{Sr}_{0.4} \mathrm{MnO}_{3}$ taken from ref. 87, where temperature independent part is subtracted as a background. (b) DMF result for $W=1 \mathrm{eV}, J_{\mathrm{H}}=1.5 \mathrm{eV}$ and $x=0.4$.

By choosing $W=1 \mathrm{eV}, J_{\mathrm{H}}=1.5 \mathrm{eV}$ and $x=0.4$, it is possible to reproduce the experimental data by the DE model. In Fig. 22(b), we show the DMF results for the semicircular DOS. The peak structure as well as its temperature dependence is in gorgement.

Let us discuss more details about the scaling relation.15,87) Experimentally, the integrated spectral weight

$$
S_{\exp } \equiv \int_{2.2 \mathrm{eV}}^{4.0 \mathrm{eV}} \mathrm{d} \omega \sigma(\omega)
$$

shows a scaling relation

$$
S_{\exp } \propto 1-M^{* 2}
$$

This is explained by the transfer of DOS by magnetic fluctuation in the DE model, as shown in eq. (63). (See also Fig. 10 in $\$ 3.3$.)

Thus, optical spectra show experimental evidence for the shift of DOS which is a typical phenomena in DE model, and give the estimate of the parameter $J_{\mathrm{H}} \sim 1.5 \mathrm{eV}$.

Summary: LSMO as a canonical DE system. As long as the high- $T_{\mathrm{c}}$ compounds are concerned, e.g. LSMO at $x=0.2 \sim 0.4$, the DE Hamiltonian accounts for various experimental data concerning the ferromagnetism and the transport

- Curie temperature. $9,16,8$, 
- Resistivity (absolute value and magnetoresistance).8.11.

- Spin and charge excitation spectra.11,12

- Scaling behaviors in charge and spin dynamics.14, 15

The mechanism of $\mathrm{MR}$ in the single-crystal of these high $T_{\mathrm{c}}$ compeunds are understood from double-exchange alone, namely due to spin disorder scattering. 6, 17, 43, 44, \&8

There still exist controversies with some experiments and the DE model. An example is the loss of spectral weight near the Fermi_level observed by photoemission experiments, 40, 89) the other is the optical conductivity spectrum 99 ) at $\omega \lesssim 1 e V$ which shows substantial loss of the Drude weight (integrated oscilator strength in the low frequency region) in contradiction with the specific heat behavior which does not show such a large mass enhancement. It should be noted that these experiments are highly sensitive to surface states, and there always remains an open question whether these experiments really measure the bulk states or are merely observing the surface states. Recently, Takenaka et al.91) reported that the optical spectrum of the "clean" surface prepared by cleaving shows larger Drude part compared to those prepared by surface filing, and is consistently large with respect to the specific heat estimate. Although the issue is still far from conclusive results, we should be very careful about such experimental details.

Let us discuss the value of $J_{\mathrm{H}}$ in manganites estimated from these calculations. From experiments concerning spin and charge dynamics, data fitting gives $J_{\mathrm{H}}=1.5 \sim 2 \mathrm{eV}$. On the other hand, static and low frequency experiments such as $T_{\mathrm{c}}$ and $\rho(T)$ measurements are well reproduced by $J_{\mathrm{H}}=3 \sim 5 \mathrm{eV}$. Such a change in the value of $J_{\mathrm{H}}$ may be understood by the renormalization of interaction. While high energy experiments (such as $\sigma(\omega)$ ) directly measure the bare value of $J_{\mathrm{H}}$, low frequency measurement is affected by the dressed quasiparticle involving the vertex renormalization. In this case, particularly, the effect of Coulomb interaction should be important. Since our DE Hamiltonian does not involve on-site Coulomb repulsion terms, elimination of the double occupancy of itinerant electrons are underestimated. This is compensated by increasing the value of $J_{\mathrm{H}}$ which also energetically prohibits the on-site double occupancy. Thus the vertex renormalization due to Coulomb repulsion may increase the value of $J_{\mathrm{H}}$ in the low-frequency scale.

Discussion: Absence of polaronic behaviors in LSMO. Millis et al. ${ }^{\text {G) }}$ discussed the discrepancies in DE model and experiments of LSMO with respect to (i) Curie temperature $\left(T_{\mathrm{c}}\right)$, (ii) temperature dependence of resistivity $(\rho(T))$, and (iii) absolute value of resistivity $\left(\rho\left(T \sim T_{\mathrm{c}}\right)\right)$. They concluded that DE alone does not explain the MR of LSMO.

What we have shown here is, however, that the accurate calculation of the DE model and the measurements of high quality crystal samples indeed give consistent results. Polaron effects, if any, should be small enough to be irrelevant. For example, Röder et al. 22 discussed the decrease of $T_{\mathrm{c}}$ due to the reduction of electron hopping caused by the polaronic effect. Thus, if polaronic interaction is included to the DE model hamiltonian, $T_{\mathrm{c}}$ of the model becomes inconsistently small compared to the experimental value.

Another point to be discussed is the experimental difference between LSMO and LCMO (see also 5 5.1). Since LCMO shows different behaviors in resistivity, e.g. temperature dependence of $\rho(T)$ as well as the MR scaling relations, different physics must be taking place. If one assumes the polaronic arguments, crossover from metallic (LSMO) to insulating (LCMO) behavior with respect to the resistivity above $T_{\mathrm{c}}$ could be understood in a following way. Insulating behavior might be due to small-polaron formation in systems with large electron-lattice coupling, while systems with smaller electron-lattice coupling might show large-polaron behaviors.

However, this argument is in contradiction with experimental data. Pair distribution function (PDF) measurement of atomic displacements observed by inelastic neutron scattering 93 shows that there exist large Jahn-Teller distortions in a dynamic way even in the metallic phase of LSMO above $T_{\mathrm{c}}$. As long as high $T_{\mathrm{c}}$ compound LSMO is concerned, lattice distortion does not seem to affect transport properties as well as magnetic behaviors $\left(e . g ., T_{\mathrm{c}}\right)$. Since large lattice 
distortion is observed both in LSMO and LCMO, 94.95 polaronic discussion alone is not sufficient to explain the qualitative difference in $\rho(T)$.

\subsection{CMR effects in low Curie temperature compounds}

What is the origin of the difference in resistivity behavior of LCMO compared to LSMO? As shown in $\S 5.2$, the DE model is sufficient to explain LSMO, and should be a good reference model to discuss LCMO. We discuss the half-metallic behavior in ferromagnetic states and the competition between ferromagnetic metals and charge ordered insulators.

Theories of CMR based on the change in transfer integral. Several theories based on microscopic models have been proposed to explain the CMR phenomena in LCMO. Polaron effects of Jahn-Teller distortions are introduced to explain the metal-insulator transition from the point of view of large polaron to small polaron crossover by magnetism.92, 96, 97) The idea of Anderson localization dwe to spin disorder as well as diagonal charge disorder has also been discussed 34, 88, 98, 99, $100,101,102$

Many of these proposals relate magnetism and transport through the change of the hopping matrix element

$$
t_{\mathrm{eff}}(T, H) \propto \cos (\theta / 2),
$$

where $\theta$ is the angle between nearest neighbor spins, as discussed by Anderson and Hasegawa. . Namely, in general, these scenarios discuss the existence of the critical value of hopping $t_{c}$. Change of the mean magnetic structure controls the value of $t_{\text {eff }}$, and it is considered that some kind of transition occurs at $t_{\mathrm{eff}}=t_{\mathrm{c}}$.

In a small polaron scenario, there exists a competition between the electron kinetic energy $E_{\text {kin }} \propto t_{\text {eff }}$ and the polaron energy $E_{\text {pol }}(\propto \lambda)$. It is characterized by the critical value for hopping $t_{\mathrm{c}}$ where transition from small polaron state (small $t_{\text {eff }}$ region) and large polaron state (large $t_{\text {eff }}$ region) occurs. Such a change in the behavior of carriers affect the conductivity through the self trapping mobility or the percolation of localized carriers. In the context of Anderson localization, critical value $t_{\mathrm{c}}$ is determined by the relationship between the mobility edge and the Fermi level. In either cases, electron hopping $t_{\text {eff }}$ is considered to be controlled by the magnetic structure through the Anderson-Hasegawa's DE mechanism. In the presence of the competition between extended and localized states, metallic states are realized at the large hopping region $t_{\mathrm{eff}}(T, H)>t_{\mathrm{c}}$, and the system becomes insulating at small hopping region $t_{\mathrm{eff}}(T, H)<t_{\mathrm{c}}$.

However, in order to explain the experiments for resistivity, these scenario need additional explanations to justify themselves. Let us point out two issues here:

- Does the transition point $t_{\mathrm{c}}$ really exist at the well-doped system $x=1 / 3 \sim 1 / 2$ ?

- Why does the "metal-insulator" transition occur only in the vicinity of $T_{\mathrm{c}}$, irrespective of carrier concentration and bandwidth?

Localization phenomena in low carrier concentration is explained by the suppressed overlaps of wavefunctions. For example, in a low carrier small polaron system, a self-trapped polaron has a short confinement length scale compared to mean polaron-polaron distances. On the other hand, if we consider the region $x \sim 1 / 3$, every two sites out of total six neighbors in a cubic lattice is occupied by other polarons. In such a high density system, quantum mechanical overlaps of polaron wavefunctions have to be quite large. In other words, in order to assume a localized state, the gain of self-trapping potential energy has to be unrealistically large to compensate the loss of kinetic energy by such a localization with small length scale. For example, dynamic Jahn-Teller scenario requires that the polaron binding energy be larger than the electron kinetic energy to make insulating behaviors for the carrier doped case. 97 ) Thus, it is difficult to understand whether the localized state really exists in a realistic parametrization of the Hamiltonian. 
Another point which is hard to explain is the fact that the metal-insulator transition always occurs in the vicinity of $T_{\mathrm{c}}$ for various A-site substitutions in both ionic radius and average valence changes. In order to explain the metal-insulator transition scenarios require the "pinning" of the critical point at $T \sim T_{\mathrm{c}}$, i.e., irrespective of carrier concentration as well as A-site bandwidth and randomness, $t_{\mathrm{c}} \sim t_{\mathrm{eff}}\left(T=T_{\mathrm{c}}\right)$ always has to be satisfied. Note that $t_{\text {eff }}$ is determined by the short range spin correlation $\left\langle S_{i} \cdot S_{j}\right\rangle$ and is a smooth and continuous function of temperature without an anomaly at $T_{\mathrm{c}}$. It is also required to explain the complete absence of the metal-insulator transition in high- $T_{\mathrm{c}}$ compounds such as LSMO at $x \sim 1 / 3$ within some realistic parametrizations.

Magnetic inhomogeneities and the nanodomain TMR mechanism. In manganites, especially in the low- $T_{\mathrm{c}}$ regions, magnetic inhomogeneities are experimentally observed. Here we discuss the importance of such inhomogeneities.

Unconventional feature in the low- $T_{\mathrm{c}}$ compounds is the presence of the central peak well below $\left.T_{\mathrm{c}}, 103\right)$ which indicates the presence of the magnetic cluster and its diffusive dynamics. From the spin diffusion constant, the correlation length of the spin clusters are estimated to be $\xi \sim 10 \AA$. Neutron elastic scattering measurements also observed the ferromagnetic cluster with correlation length $\xi \sim 20 \AA .104,105$

Magnetic inhomogeneities also show up at the linewidth of the spin wave $\Gamma_{q} \sim q^{2}$, which systematically increases as $T_{\mathrm{c}}$ is suppressed by A-site substitution. It is speculated to be due to inhomogeneity effect through spin stiffness distribution.14,15) For lower $T_{\mathrm{c}}$ compounds, there exist much prominent broadening of the spin wave dispersion at the zone boundary.106

Optical conductivity measurements 56, 107, 100, 109, 110 show that the A-site substitution causes changes in the spectrum at the peak structure around $\sim 1.5 \mathrm{eV}$ and the infrared quasi-Drude (incoherent) structures. Formation of lattice polaron at lower $T_{\mathrm{c}}$ compounds which causes $\omega \sim$ $1.5 \mathrm{eV}$ peak is discussed. Inhomogeneities in charge and magnetic structures observed by $\mu \mathrm{SR}$ and X-ray measurements 111, 112, 113) suggest that such polaronic cluster remain even at at low temperatures. Possible micrograin formation due to charge segregation as well as phase separation between ferromagnetic and antiferromagnetic domains has also been discussed.99, 50, 114)

Thus in most compounds inhomogeneous behaviors are observed experimentally. The tendency is that inhomogeneity is more prominent in the compounds with lower $T_{\mathrm{c}}$ due to smaller $\left\langle r_{\mathrm{A}}\right\rangle$ (or larger $\sigma\left(r_{\mathrm{A}}\right)$ as well).

Let us now focus on the case $x \sim 1 / 3$. As discussed in $\$ 5.1$, decrease of $T_{\mathrm{c}}$ is much larger than the estimate from the reduction of the bandwidth by $\left\langle r_{\mathrm{A}}\right\rangle$. Inhomogeneity may be playing an important role to this behavior. Since wide bandwidth compound LSMO shows a ferromagnetic metal phase while narrow band compound PCMO is a charge-ordered insulator, there exist a competition as well as a bistability of these phases in the intermediate bandwidth region. Inhomogeneous behaviors in LCMO suggests that coexistence of microscopic domains with ferromagnetic and charge-ordering correlations might happen. In the paramagnetic phase, neither correlations are long-ranged in a macroscopic sense. They should be short ranged and/or dynamic.

In such nanodomain structures with microscopic phase separation, magnetic phase transition occurs when inter-domain correlations become long ranged. Although intra-domain correlations may begin at higher temperature determined by the DE mechanism, true long range order is controlled by the domain-domain interactions mediated by the junction structures. Intermediate region with charge ordering reduces the magnetic coupling between ferromagnetic nanodomains. Then $T_{\mathrm{c}}$ should be substantially reduced. Such nanodomain structure creates two different energy scales for magnetisms, i.e. intra-domain and inter-domain interactions.

It also shows up in two correlation lengths. One is the intra-domain correlation length, which becomes the domain size at low temperature regions. The other is the inter-domain correlation length which is the length scale to determine the nature of the magnetic phase transition. It has been reported that in the low $T_{\mathrm{c}}$ compounds the magnetic correlation length does not diverge at $T_{\mathrm{c}}$ but stays constant, e.g. $\sim 20 \AA$ for NSMO 105 This unconventional behavior is understood if the typical length scale of the nanodomain structure is $\sim 20 \AA$. Due to the resolution of the 
triple-axis experiment, it seems that the macroscopic correlation length which should diverge at $T_{\mathrm{c}}$ was not detected.

Now we discuss the relationship with CMR phenomena. In the presence of nanodomain structures with ferromagnetic metals and (charge ordered) insulators, several mechanisms create magnetoresistance. A possible mechanism is the percolation of metallic nanodomains. If the external magnetic field is applied, the system gains energy by increasing the volume fraction of the ferromagnetic nanodomains. At the percolation threshold, there exists a metal-insulator transition. This scenario is, however, unlikely in the sense that metal-insulator transition occurs only at the percolation threshold, while the CMR phenomena is widely observed for various composition range away from some critical point.

Another possibility to be discussed here is the nanodomain TMR phenomena, as is commonly the case for polycrystals.68 At $T \sim T_{\mathrm{c}}$, the intra-domain spin correlation is well developed so each nanodomain can behave as a half-metallic domains. Then, application of magnetic field controls the spin valve transports between nanodomains. This scenario is most likely in the sense that it naturally explains generic MR behavior at $T \sim T_{\mathrm{c}}$ and does not assume any critical points. The conductivity is controlled by magnetism through the spin valve channels. Huge sensitivity to the external field is due to the fact that each metallic nanodomain already forms a ferromagnetic cluster. The idea is consistent with the phenomenological explanation of resistivity in LCMO by the Two-fluid model proposed by Jaime and Salomon.115) They discuss the coexistence of a metallic conductivity path and an activation-type polaronic conductivity.

Let us finally mention the origin of such nanodomain structures. Instability of electronic phase separation ${ }^{16)}$ is a candidate for the initial driving force for such phenomena, which is stabilized to form droplet structures due to long range Coulomb interactions. Another possibility is the effect of static potential disorder due to A-site cation $R^{3+}-A^{2+}$ distributions which causes charge inhomogeneities, 116$)$ as well as self-trapping effect of lattice polarons.

\section{SUMMARY AND CONCLUDING REMARKS}

In this article we discussed the thermodynamics of the DE model. We treated the model at finite temperature non-perturbatively with respect to the spin fluctuations. Various thermodynamic quantities including magnetic and transport properties are calculated. Modifications to the mean-field type treatment by Anderson-Hasegawa and de Gennes are made.

These new results are found to be very important in comparing the experimental data for manganites with the DE model. As long as the high $T_{\mathrm{c}}$ compounds (e.g. LSMO) in the metallic phase are concerned, the DE model accounts for various experimental properties including magnetic transition and resistivity. Comparison with respect to low $T_{\mathrm{c}}$ compounds (e.g. LCMO) are also discussed.

There still remains many open qyestions. One is the roles of other interactions such as lattice distortion 6 or orbital fluctuations.73, 117 It is experimentally clear that these are quite important and indeed make some long range orderings in the insulating phases. However, as we have shown in this article, such interactions does not show up in thermodynamic properties in the high $T_{\mathrm{c}}$ metallic phases. It is quite interesting how and why such "screening" of interactions occur in the metallic phase.

In other words, if we start from a realistic model for manganites with orbital and lattice degrees of freedom as well as Coulomb interactions etc., we somehow end up with the double-exchange model in the metallic phase as a consequence of neglecting high-energy excitations. Therefore, we should always regard the model as the renormalized model with parameters $t=t_{\text {eff }}$ and $J=J_{\text {eff }}$ in eq. (3). Or, in a strict sense, we should consider the action of the double-exchange model in eq. (9) with renormalized Green's function $G_{\text {eff }}$ and the coupling strength $J_{\text {eff }}$. Such a detailed renormalization studies will help us to understand other intermediate phases.

Another point of interest is to understand the nature of the inhomogeneous systems with nanodomain structures. Such study with respect to both microscopic and mesoscopic length scale 
might help us to understand the generic features of the physics in strongly correlated oxides.

\section{REFERENCES}

[1] C. Zener, Phys. Rev. 82, 403 (1951).

[2] P. W. Anderson and H. Hasegawa, Phys. Rev. 100, 675 (1955).

[3] P. G. de Gennes, Phys. Rev. 118, 141 (1960).

[4] K. Chahara, T. Ohno, M. Kasai, and Y. Kozono, Appl. Phys. Lett. 63, 1990 (1993).

[5] S. Jin et al., Science 264, 413 (1994).

[6] A. J. Millis, P. B. Littlewood, and B. I. Shrainman, Phys. Rev. Lett. 74, 5144 (1995).

[7] A. P. Ramirez, J. Phys. CM9, 8171 (1997).

[8] N. Furukawa, J. Phys. Soc. Jpn. 63, 3214 (1994).

[9] N. Furukawa, J. Phys. Soc. Jpn. 64, 2754 (1995).

[10] N. Furukawa, J. Phys. Soc. Jpn. 64, 2734 (1995).

[11] N. Furukawa, J. Phys. Soc. Jpn. 64, 3164 (1995).

[12] N. Furukawa, J. Phys. Soc. Jpn. 65, 1174 (1996).

[13] N. Furukawa, J. Phys. Soc. Jpn. 66, 2523 (1997).

[14] N. Furukawa and K. Hirota, Physica B 241-243, 780 (1998).

[15] N. Furukawa, Y. Moritomo, K. Hirota, and Y. Endoh, cond-mat/9808076.

[16] S. Yunoki et al., Phys. Rev. Lett. 80, 845 (1998).

[17] K. Kubo and N. Ohata, J. Phys. Soc. Jpn. 33, 21 (1972).

[18] Th. Pruschke, M. Jarrell, and J. Freericks, Advs. in Phys. 44, 187 (1995).

[19] A. Georges, G. Kotliar, W. Krauth, and M. J. Rozenberg, Rev. Mod. Phys. 68, 13 (1996).

[20] W. Metzner and D. Vollhardt, Phys. Rev. Lett. 62, 324 (1989).

[21] E. Müller-Hartmann, Z. Phys. B74, 507 (1989).

[22] G. Möller, A. E. Ruckenstein, and S. Schmitt-Rink, Phys. Rev. B46, 7427 (1992).

[23] Th. Pruschke, D. L. Cox, and M. Jarrel, Europhys. Lett. 21, 593 (1993).

[24] Th. Pruschke, D. L. Cox, and M. Jarrel, Phys. Rev. B47, 3553 (1993).

[25] A. Khurana, Phys. Rev. Lett. 64, 1990 (1990).

[26] H. Schweitzer and G. Czycholl, Phys. Rev. Lett. 67, 3724 (1991).

[27] M. J. Rozenberg, G. Kotliar, and X. Y. Zhang, Phys. Rev. B49, 10181 (1994).

[28] U. Brandt and C. Mielsch, Z. Phys. B 75, 365 (1989). 
[29] Q. Si, G. Kotliar, and A. Georges, Phys. Rev. B46, 1261 (1992).

[30] P. G. J. van Dongen, Phys. Rev. B45, 2267 (1992).

[31] A. Georges and G. Kotliar, Phys. Rev. B45, 6479 (1992).

[32] M. Jarrel and Th. Pruschke, Z. Phys. B90, 187 (1993).

[33] O. Sakai and Y. Kuramoto, Solid State Commun. 89, 307 (1994).

[34] C. M. Varma, Phys. Rev. B54, 7328 (1996).

[35] J. E. Hirsch, D. J. Scalapino, R. L. Sugar, and R. Blankenbecler, Phys. Rev. Lett. 47, 1628 (1981).

[36] J. E. Hirsch, R. L. Sugar, D. J. Scalapino, and R. Blankenbecler, Phys. Rev. B26, 5033 (1982).

[37] K. Kubo, J. Phys. Soc. Jpn. 33, 929 (1972).

[38] R. de Groot, Phys. Rev. Lett. 50, 2024 (1983).

[39] V. Yu Irkhin and M. I. Katsnel'son, Physics Uspekhi 37, 659 (1994).

[40] J.-H. Park et al., Nature 392, 794 (1998).

[41] J.-H. Park et al., Phys. Rev. Lett. 81, 1353 (1998).

[42] J. Sun et al., Appl. Phys. Lett. 69, 3266 (1996).

[43] T. Kasuya, Prog. Theo. Phys. 16, 58 (1956).

[44] M. E. Fisher and J. Langer, Phys. Rev. Lett. 20, 665 (1968).

[45] J. Riera, K. Hallberg, and E. Dagotto, Phys. Rev. Lett. 79, 713 (1997).

[46] M. Kagan, D. Khomskii, and M. V. Mostovoy, cond-mat / 9804213.

[47] D. P. Arovas, G. Gomez-Santos, and F. Guinea, cond-mat/9805399.

[48] E. L. Nagaev, Physica B 230-232, 816 (1997).

[49] G. Allodi et al., Phys. Rev. B56, 6036 (1997).

[50] M. Hennion et al., Phys. Rev. Lett. 81, 1957 (1998).

[51] T. A. Kaplan and S. D. Mahanti, J. Phys. Cond. Mat. 9, L291 (1997).

[52] P. Schiffer, A. Ramirez, W. Bao, and S.-W. Cheong, Phys. Rev. Lett. 75, 3336 (1995).

[53] A. Urushibara et al., Phys. Rev. B51, 14103 (1995).

[54] Y. Tomioka et al., Phys. Rev. B53, 1689 (1996).

[55] H. Hwang et al., Phys. Rev. Lett. 75, 914 (1995).

[56] Y. Moritomo, H. Kuwahara, and Y. Tokura, J. Phys. Soc. Jpn. 66, 556 (1997).

[57] P. Radaelli et al., Phys. Rev. B56, 8265 (1997).

[58] L. M. Rodriguez-Martinez and J. P. Attfield, Phys. Rev. B54, 15622 (1996). 
[59] S.-W. Cheong (unpublished).

[60] G. J. Snyder et al., Phys. Rev. B53, 14434 (1996).

[61] Y. Tomioka, A. Asamitsu, Y. Moritomo, and Y. Tokura, J. Phys. Soc. Jpn. 64, 3626 (1995).

[62] H. Yoshizawa, H. Kawano, Y. Tomioka, and Y. Tokura, J. Phys. Soc. Jpn. 65, 1043 (1996).

[63] E. O. Wollan and W. C. Koehler, Phys. Rev. 100, 545 (1955).

[64] J. B. Goodenough, Phys. Rev. 100, 564 (1955).

[65] H. Kawano et al., Phys. Rev. Lett. 78, 4253 (1997).

[66] T. Akimoto et al., Phys. Rev. B57, 5594 (1998).

[67] H. Kuwahara et al., Phys. Rev. B56, 9386 (1997).

[68] H. Hwang, S.-W. Cheong, N. Ong, and B. Batlogg, Phys. Rev. Lett. 77, 2041 (1996).

[69] A. Gupta et al., Phys. Rev. B54, 15629 (1996).

[70] P. Raychaudhuri et al., cond-mat/9807084, to be published in Phys. Rev. B1, Feb. (1999).

[71] K. Steenbeck et al., Appl. Phys. Lett. 71, 968 (1997).

[72] N. D. Mathur et al., Nature 387, 266 (1997).

[73] D. Khomskii and G. Sawatzky, Solid State Comm. 102, 87 (1997).

[74] N. Hamada, H. Sawada, and K. Terakura, in Proc. 17th Taniguchi International Conference, edited by A. Fujimori and Y. Tokura (Springer Verlag, Berlin, 1995).

[75] W. E. Pickett and D. J. Singh, Phys. Rev. B55, 8642 (1996).

[76] D. A. Papaconstantopoulos and W. Pickett, Phys. Rev. B, 12751 (1998).

[77] Y. Tokura et al., J. Phys. Soc. Jpn. 63, 3931 (1994).

[78] H. Röder, R. Singh, and J. Zang, Phys. Rev. B56, 5084 (1997).

[79] C. W. Searle and S. T. Wang, Canad. J. Phys. 47, 2703 (1969).

[80] M. F. Hundley et al., Appl. Phys. Lett. 67, 860 (1995).

[81] A. Asamitsu, Y. Moritomo, and Y. Tokura, Phys. Rev. B53, 2952 (1996).

[82] T. G. Perring et al., Phys. Rev. Lett. 77, 711 (1996).

[83] M. Martin et al., Phys. Rev. B53, 14285 (1996).

[84] A. Moudden, L. Vasiliu-Doloc, L. Pinsard, and A. Revcolevschi, Physica B 241-243, 276 (1998).

[85] L. Vasiliu-Doloc et al. (unpublished).

[86] X. Wang, Phys. Rev. B57, 7427 (1998).

[87] Y. Moritomo et al., Phys. Rev. B56, 5088 (1997).

[88] E. L. Nagaev, Phys. Rev. B58, 816 (1998). 
[89] D. Sarma, Phys. Rev. B53, 6873 (1996).

[90] Y. Okimoto et al., Phys. Rev. Lett. 75, 109 (1995).

[91] K. Takenaka et al. (unpublished).

[92] H. Röder, J. Zang, and A. R. Bishop, Phys. Rev. Lett. 76, 1356 (1996).

[93] D. Louca et al., Phys. Rev. B56, 8475 (1997).

[94] P. Dai et al., Phys. Rev. B54, R3694 (1996).

[95] S. J. L. Billinge et al., Phys. Rev. Lett. 77, 715 (1996).

[96] A. J. Millis, B. I. Shrainman, and R. Mueller, Phys. Rev. Lett. 77, 175 (1996).

[97] A. J. Millis, R. Mueller, and B. I. Shraiman, Phys. Rev. B54, 5405 (1996).

[98] E. Müller-Hartmann and E. Dagotto, Phys. Rev. B54, 6819 (1996).

[99] R. Allub and B. Alascio, Sol. State Comm. 99, 613 (1996).

[100] Q. Li, J. Zang, A. R. Bishop, and C. Soukoulis, Phys. Rev. B56, 4541 (1997).

[101] L. Sheng, D. Xing, D. Sheng, and C. Ting, Phys. Rev. B56, 7053 (1997).

[102] E. Kogan and M. Auslender, cond-mat / 9807069.

[103] J. Lynn et al., Phys. Rev. Lett. 76, 4046 (1996).

[104] J. D. Teresa et al., Phys. Rev. B54, 12689 (1996).

[105] J. Fernandez-Baca et al., Phys. Rev. Lett. 80, 4012 (1998).

[106] H. Hwang et al., Phys. Rev. Lett. 80, 1316 (1998).

[107] S. Kaplan et al., Phys. Rev. Lett. 77, 2081 (1996).

[108] J. H. Jung et al., Phys. Rev. B57, 11043 (1998).

[109] M. Quijada et al., cond-mat/9803201.

[110] A. Machida, Y. Moritomo, and A. Nakamura, Phys. Rev. B58, 4281 (1998).

[111] R. H. Heffner et al., Phys. Rev. Lett. 77, 1869 (1996).

[112] S. Yoon et al., Phys. Rev. B58, 2795 (1998).

[113] C. H. Booth et al., Phys. Rev. B57, 10440 (1998).

[114] T. Perring, G. Aeppli, Y. Moritomo, and Y. Tokura, Phys. Rev. Lett. 78, 3197 (1997).

[115] M. Jaime and M. Salamon (unpublished).

[116] C. M. Varma (unpublished).

[117] S. Ishihara, J. Inoue, and S. Maekawa, Physica C 263, 130 (1996). 